\title{
Review Article \\ The Sarcomeric M-Region: A Molecular Command Center for Diverse Cellular Processes
}

\author{
Li-Yen R. Hu, Maegen A. Ackermann, and Aikaterini Kontrogianni-Konstantopoulos
}

Department of Biochemistry and Molecular Biology, School of Medicine, University of Maryland, 108 N Greene Street, Baltimore, MD 21201, USA

Correspondence should be addressed to Aikaterini Kontrogianni-Konstantopoulos; akons001@umaryland.edu

Received 17 November 2014; Accepted 8 February 2015

Academic Editor: Oleg S. Matusovsky

Copyright (C) $2015 \mathrm{Li}$-Yen R. Hu et al. This is an open access article distributed under the Creative Commons Attribution License, which permits unrestricted use, distribution, and reproduction in any medium, provided the original work is properly cited.

\begin{abstract}
The sarcomeric M-region anchors thick filaments and withstands the mechanical stress of contractions by deformation, thus enabling distribution of physiological forces along the length of thick filaments. While the role of the M-region in supporting myofibrillar structure and contractility is well established, its role in mediating additional cellular processes has only recently started to emerge. As such, M-region is the hub of key protein players contributing to cytoskeletal remodeling, signal transduction, mechanosensing, metabolism, and proteasomal degradation. Mutations in genes encoding M-region related proteins lead to development of severe and lethal cardiac and skeletal myopathies affecting mankind. Herein, we describe the main cellular processes taking place at the M-region, other than thick filament assembly, and discuss human myopathies associated with mutant or truncated M-region proteins.
\end{abstract}

\section{Introduction}

The M-band is a dense protein-packed structure at the center of the A-band of cardiac and skeletal muscle cells (Figure 1 and Table 1). Under the electron microscope, M-band appears as a series of dark transverse lines spanning $500-750 \AA$, depending on fiber type and species [1]. Named for the German word "mittelscheibe," which means "central disc," the M-band lies at the center of the bare zone, which is devoid of myosin heads and cross-bridges [2] but encompasses overlapping arrays of antiparallel myosin rods [3]. Adjacent myosin rods are connected via M-bridges, forming a regular hexagonal lattice [4]. The M-bridges are necessary to maintain thick filament alignment and aid in the controlled distribution of mechanical stress across the sarcomere during active contraction [5].

In addition to the rod region of myosin, the M-band is "home" to several other proteins (Figure 1 and Table 1). As such, the $\mathrm{COOH}$-termini of titin molecules from half sarcomeres converge in an antiparallel fashion at the Mband [6]. Composed of immunoglobulin (Ig) domains and unique sequences, the $\mathrm{COOH}$-terminus of titin is located downstream of its kinase domain, which is found at the junction of A- and M-bands.

The M-band also contains myomesin, M-protein, and myomesin-3, which share similar domain architectures and are primarily composed of Ig and fibronectin type III (FnIII) domains, but contain distinct $\mathrm{NH}_{2}$-terminal heads $[7,8]$. These proteins are the principal components of M-bridges forming the backbone of the M-band filamentous system, which cross-links neighboring thick filaments $[6,8]$.

Also localizing at the level of the M-region are additional sarcomeric and membrane associated proteins, including obscurins, select variants of Myosin Binding Protein-C Slow, ankyrins, and spectrins [9-12]. These contribute to the assembly and stabilization of the M-region and its linkage with the sarcomeric cytoskeleton, the sarcoplasmic reticulum (SR), and the sarcolemma [13].

Within the last decade, our knowledge of the sarcomeric M-region has steadily expanded. To date, there are several excellent reviews on protein complexes mediating the assembly and organization of the entire M-region encompassing the M-band core as well as its periphery, and its role in thick filament assembly and integration into A-bands $[5,14]$. 
TABle 1: Properties of M-region proteins.

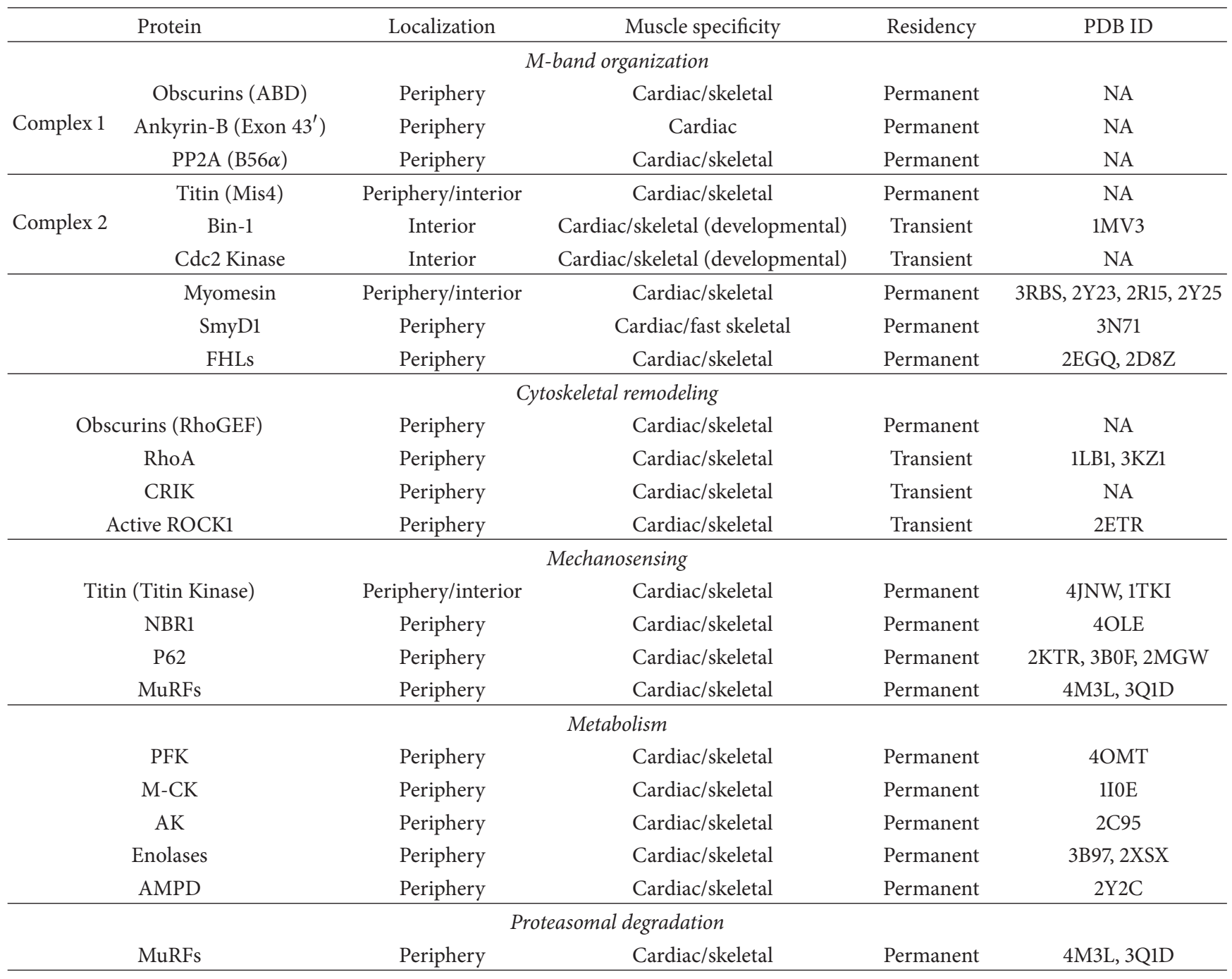

Note: protein domains mediating complex formation or participating in cellular processes are shown in parenthesis when known. Acronyms of proteins are described in the text; ABD: ankyrin binding domain; NA: not available. The PDB files of proteins in Complex 1 and Complex 2, as well as obscurins (RhoGEF) and titin (titin kinase), are associated with the specific domains that mediate binding within the complex; in all other cases, the available PDB files for the entire protein are provided.

Herein, we focus on key protein mediators of additional cellular processes occurring at the M-region. In addition, we highlight skeletal and cardiac myopathies that are linked to mutations in genes encoding M-region related proteins.

\section{Cellular Processes at the M-Region}

M-region is the hub for multiple cellular processes including signal transduction, metabolism, mechanosensing, and proteasomal degradation. Such processes support cellular homeostasis, myofibrillar organization, and contractile activity by maintaining sarcomeric integrity, meeting the energy demand during active contraction, and enabling adaptation to different biochemical and biomechanical stimuli. Below we will address these important processes occurring at the Mregion and discuss key proteins.
2.1. Signal Transduction via Posttranslational Modifications. Two main types of posttranslational modifications, phosphorylation and sumoylation, have been described at the Mregion. These mediate proper protein localization, regulate protein-protein interactions, and relay signals in response to biochemical or biomechanical stimuli.

2.1.1. Phosphorylation. Several M-region proteins possess active kinase domains and/or are regulated by phosphorylation. Below we discuss such proteins.

Titin ( 3-4 MDa). The giant protein titin extends longitudinally across a half-sarcomere, with its $\mathrm{NH}_{2}$-terminus anchored to the $\mathrm{Z}$-disc, and its $\mathrm{COOH}$-terminus localized at the center of the M-band [15]. The M-band portion of titin $(\sim 200 \mathrm{kDa})$ is composed of ten Ig CII type domains, which are interspersed with unique nonmodular segments, termed 


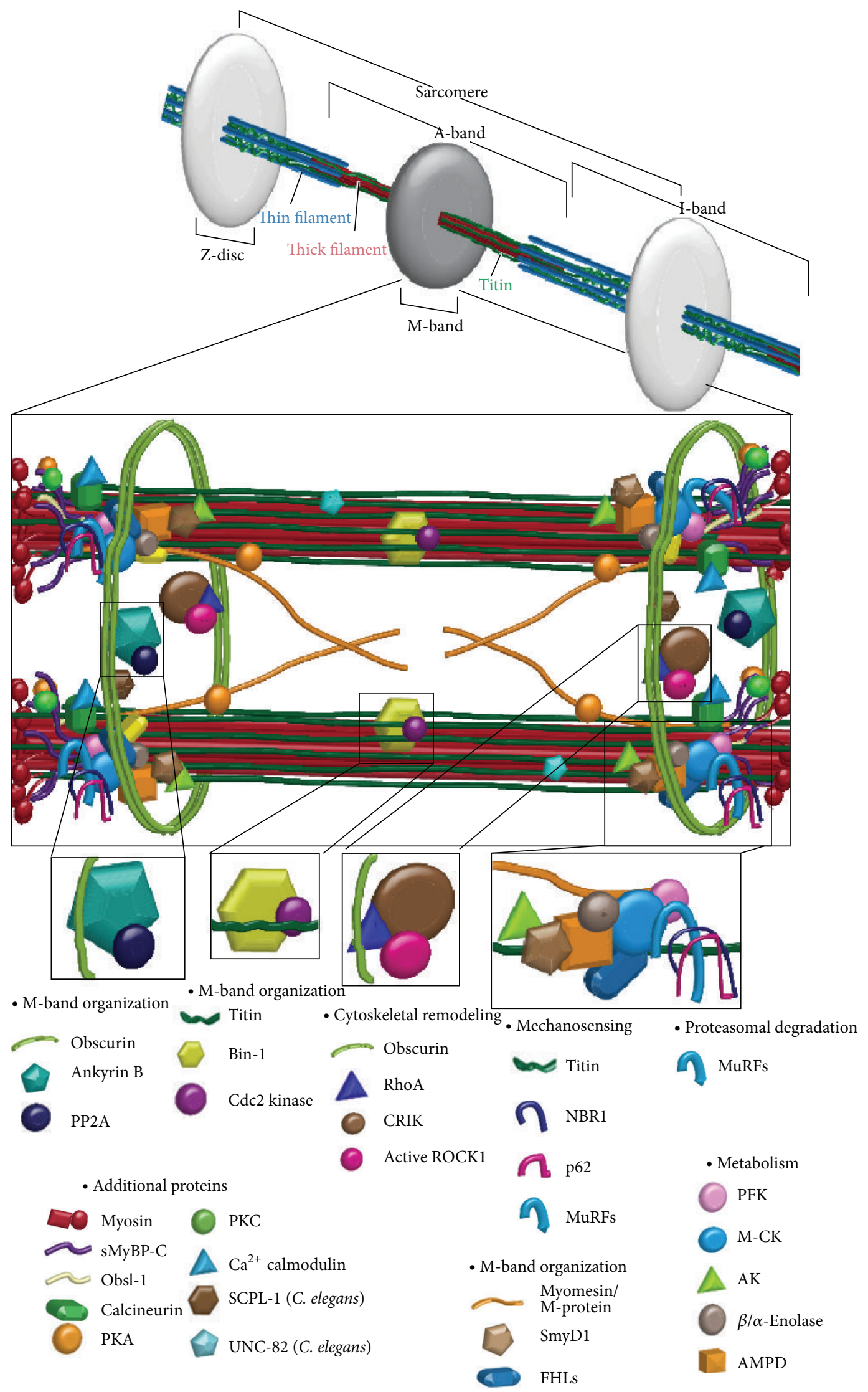

FIGURE 1: Sarcomeric M-region road map and cellular processes. Schematic representation of the sarcomeric M-region depicting key proteins and highlighting cellular processes. 
M-insertions $[13,16,17]$. The insertion between Ig CII-5 and Ig CII-6 contains tandem lysine-serine-proline (KSP) repeats, which are heavily phosphorylated by Cdc2 kinase in developing, but not in differentiated muscle cells [16]. The phosphorylated KSP repeats interact in vitro with the $\mathrm{SH} 3$ domain of the tumor suppressor bridging integrator protein 1 (Bin1) [18]. Bin1 is a negative regulator of c-Myc activation that is preferentially expressed in differentiating, but not mature, myotubes [18]. Transgenic mice overexpressing the $\mathrm{SH} 3$ domain of Binl exhibit dramatic disarray in myofiber size and structure [18]. Conversely, mouse C2C12 skeletal myoblasts fail to differentiate following downregulation of Bin1 $[19,20]$, and Binl homozygous knock-out mice develop hypertrophic cardiomyopathy leading to perinatal lethality, although skeletal muscles do not exhibit any apparent abnormalities at this stage [21]. It is therefore possible that disruption of the interaction between the phosphorylated KSP repeats of titin and Binl at the M-band may result in deregulated myofibrillar assembly, loss of differentiation, and aberrant fiber size.

In addition to being phosphorylated within its M-band portion, titin may regulate different sarcomeric processes via its kinase domain, residing at the periphery of the M-band $[6,13,22,23]$; one of these processes is mechanosensing, which will be discussed in a later section of this review.

Obscurin (720-890 kDa). Incorporated early during development into the periphery of the sarcomeric M-band, obscurin is an important player of thick filament assembly and stabilization [24]. Downregulation of obscurin using siRNA technology leads to selective disorganization of $\mathrm{M}$ - and Abands in developing skeletal and cardiac myocytes, indicating its scaffolding role [24-26]. Importantly, recent studies have demonstrated that the two Ser/Thr kinase domains, which are present at the $\mathrm{COOH}$-terminus of giant obscurin-B are catalytically active and can directly bind major components of the M-band, including titin and four and a half LIM domains 2 (FHL2) protein $[27,28]$. Examining if these proteins are catalytic substrates of the obscurin kinase domains at the Mband will be the next challenge.

Obscurin is also involved in dephosphorylation events at the M-band. Ankyrin-B (Ank-B), fulfilling its role as an anchoring protein, binds directly to both obscurin and the regulatory subunit, B56 $\alpha$, of protein phosphatase 2A (PP2A) at the M-band [29, 30]. Overexpression of the obscurin-binding region of Ank-B in isolated adult cardiomyocytes results in displacement of B56 $\alpha$ from the M-band [29]. Moreover, UNC-89, the obscurin homologue in $C$. elegans, directly binds to small C-terminal domain (CTD) phosphatase-like 1 (SCPL-1) at the M-band [28, 31]. However, the cellular processes that PP2A and SCPL-1 mediate at the M-band are still unknown.

Myomesin (178-188 kDa). Spanning the entire M-region by residing in the interior yet extending to the periphery of the M-band, myomesin serves as a cross-linker between myosin, titin, and obscurin $[32,33]$. It consists of tandem Ig and FnIII domains, along with a nonmodular $\mathrm{NH}_{2}$-terminus [32-35]. While the $\mathrm{NH}_{2}$-terminus of myomesin interacts with sarcomeric myosin [36], the region encompassing Ig domains 4 and 5 (My4-My5) directly interacts with titin Ig4 within its M-band portion (MIg4) $[32,34]$. Phosphorylation of the linker region between myomesin domains My4 and My5 by protein kinase A (PKA) abolishes its binding to titin MIg4 [32]. Importantly, the linker region also interacts with two other M-band proteins, obscurin, and obscurin-like 1, but these interactions are not modulated by phosphorylation [33]. Thus, a ternary complex of regulated and potentially constitutive interactions between myomesin and titin, and myomesin and obscurin, or obsl-1 occurs at the M-band [33].

M-Protein ( $165 \mathrm{kDa})$. Similar to myomesin, M-protein also consists of tandem Ig and FnIII domains, which are preceded by a unique $\mathrm{NH}_{2}$-terminus $[6,37,38]$. Unlike myomesin though, which is expressed ubiquitously in all striated muscles, M-protein is only expressed in cardiac and fast-twitch skeletal muscles [39-41]. Phosphorylation at S76, present in the nonmodular $\mathrm{NH}_{2}$-terminus of $\mathrm{M}$-protein, by PKA abolishes its binding to myosin [42].

Thus, the PKA-mediated phosphorylation of both myomesin and M-protein may modulate thick filament integration, organization, and stability at the M-band during myofibrillogenesis or myofilament turnover.

Myomesin-3 ( $162 \mathrm{kDa})$, also composed of a nonmodular $\mathrm{NH}_{2}$-terminus and tandem Ig and FnIII domains, is present at the M-band, too [8]. Unlike myomesin, which is ubiquitously present in all striated muscles and M-protein that is preferentially expressed in cardiac and fast-twitch skeletal muscles, myomesin-3 is selectively expressed in slow-twitch skeletal muscles [8, 43]. Whether myomesin3 is subjected to posttranslational modifications, however, remains to be investigated. Along the same lines, select variants of Myosin binding protein-C slow (MyBP-C slow), FHL1, and FHL2 and obscurin-like 1 also localize at the Mregion [10, 33, 44-48]. Out of these proteins, at least MyBP$\mathrm{C}$ slow is modulated via phosphorylation mediated by PKA and PKC [49]. Moreover, the $\mathrm{Ca}^{2+} /$ calmodulin-dependent serine/threonine phosphatase, calcineurin (also known as protein phosphatase $2 \mathrm{~B}, \mathrm{PP} 2 \mathrm{~B}$ ), is also present at the $\mathrm{M}$ band and may mediate dephosphorylation events of $\mathrm{M}$ band proteins [47]. At this time though, the physiological significance of the phosphorylation and dephosphorylation events involving these proteins remains speculative.

In addition to the phosphorylation events discussed above, which have been mainly studied in mammalian striated muscles, additional protein players involved in posttranslational modification events within the M-region have been reported in C. elegans. Unlike the cross-striated muscles of mammals, myofilaments of body muscles in C. elegans are arranged in oblique striations. Therefore, both the protein composition and the functional role(s) of individual proteins at the M-region of body muscles of C. elegans may differ from those of mammalian striated muscles. Below, we provide such an example.

UNC-82 (33-203 kDa). Recently identified as a Ser/Thr kinase that localizes at or near the M-band in C. elegans, UNC-82 is closely related to mammalian proteins AMPK-related protein 
kinase 5 (ARK5) and sucrose nonfermenting AMPK-related kinase (SNARK) (also known as NUAK1 and NUAK2, resp.), which are members of the AMP-activated protein kinase (AMPK) family of Ser/Thr kinases [50]. While the enzymatic activity and substrates of UNC-82 remain to be determined, mutant C. elegans embryos containing either a missense mutation presumably abolishing its kinase activity or a nonsense mutation resulting in truncated UNC-82 exhibit disorganized A- and M-bands [50]. Interestingly, its closely related mammalian counterparts, ARK5 and SNARK, have been implicated in glucose metabolism in skeletal muscle $[51,52]$; however the localization of ARK5 and SNARK has yet to be examined.

2.1.2. SUMOylation. In addition to phosphorylation, proteins at the M-region undergo sumoylation. While less studied compared to phosphorylation, sumoylation has been implicated as a possible mechanism for targeting proteins to the M-region.

Myomesin (178-188 $\mathrm{kDa}$ ). In addition to being regulated via phosphorylation, myomesin is also subjected to sumoylation, possibly at K228, in adult rat cardiomyocytes [53]. Moreover, in neonatal rat ventricular myocytes (NRVM), where myomesin is localized to the nucleus, overexpression of the SUMO peptide mediates its translocation to the cytoplasm and promotes sarcomeric organization [54]. Sumoylation of myomesin is mediated by myofibrillogenesis regulator- 1 (MR-1), which is highly expressed in both skeletal and cardiac muscles $[54,55]$. While overexpression of MR-1 in mice enhances cardiac hypertrophy stimulated by angiotensin II, overexpression of MR-1 in NRVM induces sarcomeric organization and translocation of myomesin from the nucleus to the cytoplasm, similar to SUMO overexpression $[54,56]$. Consistent with this, downregulation of MR-1 abolishes SUMO-induced translocation of myomesin to the cytoplasm and sarcomeric organization [54].

SET and MYND Domain Containing-1 (SmyD1) ( 54$56 \mathrm{kDa}$ ). SmyD1 is another M-band protein whose localization is modulated by sumoylation $[57,58]$. Also known as Bop, SmyD1 is a histone methyltransferase that is abundantly expressed in striated muscles with its methyl transferase activity attributed to its $\mathrm{Su}(\mathrm{var}) 3-9$, enhancer-of-zeste, and trithorax (SET) domain [59-63]. In mouse, the smyd1 locus encodes two alternatively spliced isoforms, SmyD1_tvl, and SmyD1_tv2 (also known as skm-Bop1 and skm-Bop2, resp.) $[60,61,63]$. SmyD1_tv1 and SmyD1_tv2 localize at the Mband and the nucleus in both cardiac and skeletal muscles $[57,60]$. Homozygous deletion of smyd1 in mice results in embryonic lethality at E10.5 due to disrupted ventricular formation, which is accompanied by loss of right ventricles [61]. Recently, skeletal muscle- and heart-specific $\alpha$ nascent polypeptide-associated complex, skNAC, was shown to mediate the sumoylation of SmyD1 proteins and thus regulate their nuclear export and translocation to the Mband during sarcomerogenesis $[58,64]$. Thus, sumoylation mediates SmyD1 targeting to the M-band, where it potentially regulates the activities of major M-band proteins, such as myosin and muscle-type creatine kinase, via its methyl transferase activity.

Proteins at the M-region undergo additional posttranslational modifications, such as acetylation, methylation, and neddylation. For instance, small ankyrin 1.5 (sAnk1.5) is subjected to acetylation and neddylation $[65,66]$, while muscle-type creatine kinase and myosin are subjected to acetylation and methylation $[66,67]$. However, the functional significance and the identity of the relevant enzymes carrying out these posttranslational modifications at the M-band remain to be further investigated.

2.2. Cytoskeletal Remodeling via Small GTPases. Small GTPases play important roles in diverse cellular and developmental processes, including cytoskeletal remodeling, actomyosin contractility, vesicle transport, growth, and proliferation [68-72]. Small GTPases are regulated via repeating cycles of GTP binding and hydrolysis. Three accessory proteins contribute to their regulation: (i) GTPase activating proteins (GAP), which hydrolyze GTP to GDP, inactivating small GTPases, (ii) guanine nucleotide exchange factors (GEF), which mediate the exchange of GDP to GTP activating small GTPases, and (iii) guanosine nucleotide dissociation inhibitors (GDI), which prevent the exchange of GDP for GTP by sequestering GTPases and preventing them from binding to downstream effectors [73, 74]. Ras homolog gene family, member A protein, RhoA, localizes at the M-band in adult skeletal and cardiac muscles $[66,75]$. While RhoA mediates several cellular processes, the discussion below will mainly focus on its roles in cytoskeletal remodeling at the M-band.

2.2.1. RhoA Signaling in Skeletal Muscle. Inactive RhoA preferentially localizes to the M-band of adult skeletal myofibers, whereas active RhoA exhibits a dual distribution, at both M-bands and Z-disks [75]. RhoA activity is significantly increased following overexpression of the obscurin RhoGEF motif in adult rat tibialis anterior (TA) muscle and after injury induced by large-strain lengthening contractions [75]. Consistent with this, the RhoGEF/Pleckstrin homology cassette of Unc-89, the C. elegans obscurin homologue, activates Rho-1, the RhoA C. elegans homologue [76]. In mammalian skeletal muscle, active RhoA leads to loss of citron rhointeracting kinase (CRIK), which is involved in the regulation of cytokinesis in proliferating cells, from M- and A-bands $[75,77,78]$. Moreover, active RhoA leads to translocation of Rho-associated protein kinase 1 (ROCK1) from the Z-disk to the I-band, the Z/I junction, and the M-band [75]. Although the functional ramifications of the concurrent loss of CRIK from the M-band and the translocation of ROCK1 to the M-band are still elusive, it is likely that they mediate the activation of stretch-response genes leading to cytoskeletal remodeling or the development of hypertrophy following injury. Consistent with this, activation of the RhoA/ROCK pathway in dystrophin/utrophin double knockout mice has been implicated with heterotopic ossification, while inhibition of the RhoA/ROCK pathway has been associated with improved myogenic potential $[79,80]$. 
RhoA localizes at both M-bands and Z-disks in cardiac muscle, too, although its distribution has not been correlated with its state of activation [66]. Extensive studies have focused on the diverse processes that RhoA mediates in the developing and adult myocardium, emphasizing its roles in the regulation of actin filament assembly and stress fiber formation, sarcomeric organization, induction of a hypertrophic response, tolerance to ischemia/reperfusion, survival, and apoptosis [81-88]. Given that it is currently unknown whether RhoA contributes to these processes through its interactions at the Z-disk or the M-band, we will refrain from presenting such studies in detail.

2.3. Mechanosensing. In addition to biochemical stimuli, muscle cells respond to biomechanical stimuli by modulating protein expression through activation of signaling pathways $[89,90]$. Consistent with this, both the Z-disk $[91,92]$ and the M-band [92] contain mechanosensors that may transform biomechanical stimuli to biochemical signals. At the M-band, the kinase domain of titin is a major player mediating cell responses to mechanical stress.

2.3.1. Titin Kinase. Use of atomic force microscopy (AFM) has demonstrated that the kinase domain of titin is activated upon exertion of mechanical force, leading to unfolding of its regulatory autoinhibitory tail and phosphorylation of Y170, allowing ATP binding to the catalytic aspartate [93]. It is therefore likely that activation of the titin kinase via mechanical force may result in regulation of its proximal substrates via phosphorylation. This is exemplified in the case of the direct interaction between the titin kinase domain and the musclespecific ring finger (MuRF) complex, consisting of NBR1 (neighbor of BRCA1 gene 1 protein)/p62/MURF-2, which has been suggested to regulate protein turnover in response to mechanical force [23]. Both NBR1 and p62 are substrates of titin kinase [23] and are required in autophagosomemediated protein degradation by serving as receptors for ubiquitinated proteins [94-96]. Since NBR1 can only bind to the semiopened conformation of the titin kinase that is likely induced by exertion of mechanical force during stretching, it is possible that MuRF-2 is anchored to the M-band via the interaction of NBR1 with the kinase domain of titin [23]. Consistent with this, denervation of skeletal myofibers or mechanical arrest of neonatal cardiomyocytes leads to dissociation of the MuRF-2/NBR1/p62 complex, with MuRF2 translocating to the nucleus in both skeletal and cardiac cells where it suppresses serum response factor- (SRF-) mediated expression of hypertrophic genes, and p62 accumulating at intercalated disks in cardiac cells [23]. It is noteworthy to mention, however, that a recent study indicated that the kinase domain of titin is not enzymatically active but serves as a scaffold for other proteins that localize to the M-region [97]. Additional studies are warranted to resolve these opposing results.

2.4. Metabolism. Sarcomeric M-band is strategically located in close proximity to where significant amounts of energy are consumed during repeating cycles of actomyosin contractility
[98-100]. Maintaining the ATP and ADP levels at optimal concentrations in the sarcoplasm is essential in sustaining muscle activity, as depletion of ATP or accumulation of ADP would attenuate contractility [101]. Below we discuss several proteins localized at the M-band, which play key roles in metabolism by maintaining the ATP/ADP ratio at optimal levels within the sarcoplasm.

2.4.1. Muscle-Type Creatine Kinase (M-CK). Creatine kinase (CK) catalyzes the phosphate transfer from phosphocreatine to ADP, generating ATP and creatine (Phosphocreatine ${ }^{2-}$ $+\mathrm{MgADP}^{-}+\mathrm{H}^{+} \leftrightarrow \mathrm{MgATP}^{2-}+$ creatine) [102]. In the mammalian genome, there are four gene loci encoding the different CK isoenzymes: muscle-type CK (M-CK), braintype CK (B-CK), ubiquitous mitochondrial CK (uMtCK), and sarcomeric mitochondrial CK (sMtCK) [103]. While both $\mathrm{M}-\mathrm{CK}$ and sMtCK are readily expressed in striated muscles, only M-CK is present within the sarcomere [103-105]. M$\mathrm{CK}$ functions as a dimer and localizes at the M-band by interacting with myomesin, M-protein, and FHL-2 $[46,106]$. Homozygous null M-CK mice exhibit decreased voluntary running capability, which is accompanied by a significant reduction in force production during initial contractions due to inadequate supply of local ATP [105, 107-109]. Interestingly, malignant transformation of skeletal muscle to sarcoma results in reduction of $\mathrm{M}$-CK levels, indicating that M-CK supports the specific metabolic needs of skeletal myofibers, which gradually lose their differentiation status and contractile properties during sarcoma development [110].

2.4.2. Adenylate Kinase (AK). Similar to $\mathrm{M}-\mathrm{CK}, \mathrm{AK}$ is also present at the M-band [46]. AK catalyzes the transfer of a phosphate from ADP to another ADP to generate AMP and ATP and vice versa $\left(\mathrm{MgADP}^{-}+\mathrm{ADP}^{2-} \leftrightarrow \mathrm{MgATP}^{2-}\right.$ $+\mathrm{AMP}^{-}$), thus maintaining myofibrillar ATP and ADP concentrations at optimal levels [111, 112]. There are nine AK isoenzymes in mammals, referred to as AK1-AK9 [113]. While the majority of AKs localize to mitochondria, AK1 and AK7 are primarily present in the sarcoplasm [113]. At rest, skeletal muscles of homozygous AK1 knockout mice contain increased levels of AMP, without any other pathological phenotype [112]. However, upon induction of high frequency (90-120 tetani/min) contractions, gastrocnemius, plantaris, and soleus AK1 null muscles exhibit increased levels of ADP, accompanied by slower relaxation rate, although the magnitude of the generated force is unaltered $[112,114$, 115]. The slower relaxation rate is likely due to reduced Gibbs-free energy in response of the increased ADP : ATP ratio, resulting in compromised sarco/endoplasmic reticulum $\mathrm{Ca}^{2+}$-ATPase (SERCA) activity and decreased $\mathrm{Ca}^{2+}$ uptake by the SR [112]. Moreover, exposure of AK1 deficient mice to ischemia/reperfusion results in accelerated loss of cardiac contractility and reduced ATP and ADP levels during reperfusion, underscoring the importance of AK1 in supporting myocardial function by regulating energy metabolism [116]. The presence of AK in the sarcomere is therefore essential for meeting the high-energy demands of muscle cells and protecting them from insults. 
2.4.3. Adenosine Monophosphate Deaminase (AMPD). Three genes encoding AMPD proteins have been identified: $A M P D 1, A M P D 2$, and AMPD3 (reviewed in [117]). AMPD1 encodes an AMPD form that is preferentially expressed in striated muscles, M-AMPD [118, 119]. M-AMPD at the Mband is coupled with M-CK and AK to modulate the ATP, ADP, and AMP levels $[101,120]$. AMPD catalyzes the removal of an amine group from AMP, giving rise to ammonia and $\operatorname{IMP}\left(\mathrm{AMP}^{-} \rightarrow \mathrm{IMP}^{-}+\mathrm{NH}_{3}\right)$ [101]. In conjunction with $\mathrm{AK}$, AMPD maintains constant intracellular ADP levels, therefore preventing AMP accumulation and favoring the formation of ATP by AK [101].

2.4.4. Phosphofructokinase (PFK). The fourth metabolic enzyme residing at the $\mathrm{M}$-band, $\mathrm{PFK}$, mediates the transfer of a phosphate group from ATP to fructose-6-phosphate to yield fructose-1, 6-bisphosphate (Fructose-6- $\mathrm{P}^{-}+\mathrm{MgATP}^{2-} \rightarrow$ Fructose-1,6- $\mathrm{P}_{2}{ }^{2-}+\mathrm{MgADP}^{-}$), and is a rate-limiting enzyme of the glycolytic pathway $[121,122]$. There are three PFK isoenzymes in mammals, PFK-M (muscle), PFK-L (liver), and PFK-P (platelet). Skeletal muscles express exclusively PFK$M$, whereas cardiac muscle expresses all three isoenzymes, with PFK-M being the predominant one [123]. Fully activated PFK exists in tetrameric or a more complex oligomeric form, while dimeric PFK confers minimal activity [124]. PFK's activity is modulated by allosteric regulators (e.g., adenosine phosphates and fructose-2, 6-bisphosphate), interacting partners (e.g., F-actin and calmodulin), or posttranslational modifications (e.g., acylation and phosphorylation) [122, 124126]. Thus, AMP and ADP stabilize PFK in a tetrameric conformation, whereas ATP and citrate stabilize PFK in a dimeric conformation [121, 124, 127-132]. Enhanced binding to F-actin in response to insulin stimulation also stabilizes the tetrameric form of PFK and maintains it in an active conformation [133-135]. Alternatively, PFK may be regulated by a complex mechanism in response to calcium fluctuation in the sarcoplasm [136]. Given the presence of two calmodulin binding sites in PFK, it has been proposed that PFK activity is strongly inhibited when both sites are occupied [124, 137]. However, occupation of only one calmodulin site may abrogate the inhibitory effect mediated by ATP- and citratebinding via induction of a dimeric conformation that is fully active [124, 138]. Intriguingly, phosphorylation mediated by calmodulin-dependent kinase (CaMK) results in increased sensitivity to ATP inhibition [129]. Since calmodulin binding is modulated by $\mathrm{Ca}^{2+}$, the activity of PFK may be modulated by the $\mathrm{Ca}^{2+}$ levels in the sarcoplasm, especially during contractions. PFK-M homozygous null mice $\left(\mathrm{PFKM}^{-/-}\right)$ exhibit high mortality at weaning $(60 \%)$, reduced life-span ( 3-6 months), and decreased ATP concentration in skeletal muscles, accompanied by increased glycogen content and exercise intolerance [139]. Notably, the small number of PFKM null animals that survive beyond 6 months of age develops cardiac hypertrophy by year one [139]. The reduced viability of the PFKM deficient mice is consistent with PFK being the rate-limiting enzyme in the glycolytic pathway and underscores its key role in energy production.
2.4.5. Enolase. In addition to PFK, enolase is another glycolytic enzyme that resides at the M-band [140, 141]. Three gene loci encode the three known enolase isozymes, which are expressed in different tissues: nonneuronal enolase, $\alpha$ $(N N E)$, muscle-specific enolase, $\beta$ (MSE), and neuronalspecific enolase, $\gamma$ (NSE) $[142,143]$. Both $\alpha$ - and $\beta$-isozymes are expressed in cardiac and skeletal muscles, localize at the M-band, and may form homo- or heterodimers $[140,141,144$, 145]. Dimeric enolase converts the glycolytic intermediate 2phospho-D-glycerate to phosphoenolpyruvate (2-phosphoD-glycerate $^{2-} \leftrightarrow$ phosphoenolpyruvate $^{2-}+\mathrm{H}_{2} \mathrm{O}$ ) [146]. While $\beta \beta$-enolase homodimers are predominantly expressed in skeletal muscle, especially in Type II muscle fibers [141, $147,148], \alpha \alpha-, \alpha \beta$-, and $\beta \beta$-dimers are present in cardiac muscle $[145,148,149]$. The relative expression of $\alpha$ and $\beta$ isozymes in the heart is important in fine-tuning metabolic activity. Consistent with this, in a rat hypertrophy model induced by aortic stenosis, where the rate of glycolysis is increased [150], the ratio of the $\alpha$ - to $\beta$-isozymes is increased in the heart, due to reduced expression of $\beta$-enolase, although the levels of $\alpha$-enolase are unaltered [149]. Conversely, in the spontaneous hypertensive (SHR) rat model, the hypertrophied heart expresses increased levels of the $\alpha$-isozyme, which is also hyperphosphorylated [149, 151, 152]. While the $\alpha$ - and $\beta$-enolases exhibit comparable enzymatic kinetics, the hyperphosphorylated $\alpha$-isozyme performs slower catalysis $[152,153]$. Overexpression of $\alpha$-enolase in response to ischemia/reperfusion in a rat model confers improved contractility of affected cardiomyocytes [154]. Consistent with its protective role in the heart, $\alpha$-enolase is significantly upregulated in mouse skeletal muscle, which predominately expresses $\beta$-enolase, in response to muscle injury induced by cardiotoxin; interestingly, the expression of $\beta$-enolase is drastically decreased after the first day but recovers a week later [155]. Similarly, in rat skeletal muscles subjected to denervation, the levels of the $\alpha \alpha$ dimer are modestly increased, while the levels of the $\beta \beta$-dimer are decreased [148]. It is noteworthy to mention that since $\alpha$-enolase may also serve as a heat-shock protein or a plasminogen receptor involved in cardiac remodeling and muscle regeneration, it is possible that the protective role of $\alpha$-enolase may not only be related to its glycolytic activity $[152,156]$.

Regulation of energy metabolism may also be mediated through proteasomal degradation. Muscle-specific RING finger proteins (MuRFs) present at the M-band ubiquitinate metabolic enzymes, which are subsequently targeted for proteasomal degradation [157-159]. Along these lines, oxidized muscle-type creatine kinase is rapidly ubiquitinated by MuRF-1 and subsequently targeted to the proteasome $[159,160]$. In addition, MuRF-1 also interacts with adenylate kinase [157], although the effects of this interaction are still elusive. The importance of MuRFs at the M-band is further discussed in the following section.

\subsection{Proteasomal Degradation}

MuRFs. The MuRF family consists of three members, MuRF1, MuRF-2, and MuRF-3, which are E3 ubiquitin ligases, and preferentially expressed in striated muscles [161-163]. 
They contain a RING finger domain at their $\mathrm{NH}_{2}$-terminus and transfer ubiquitin-chains to the proteins destined for proteasomal degradation $[158,162]$. The poly-ubiquitinated proteins are recognized by the proteasome, subjected to deubiquitination, unfolding, and hydrolysis in its proteolysis core [164]. While MuRF-3 is ubiquitously expressed in skeletal and cardiac muscles, MuRF-1 is preferentially expressed in cardiac and fast-twitch skeletal muscles, and MuRF-2 is predominantly expressed in slow-twitch skeletal muscles, with minimal expression in cardiac and fast-twitch skeletal muscles $[165,166]$. All three MuRFs localize to the M-band and the nucleus [167]. Additionally, MuRF-1 and MuRF-3 are also present at the Z-disc [167].

2.5.1. MuRF-1. MuRF-1 interacts with the Ig168/Ig169 domains within the A-band portion of titin, while the presence of the adjacent titin kinase domain enhances this interaction [97, 163]. Overexpression of MuRF-1 disrupts the organization of $\mathrm{M}$ - and A-bands, but not of Z-disks and I-bands, in chick cardiomyocytes [168], indicating that increased protein turnover rate at $\mathrm{M}$ - and $\mathrm{A}$-bands results in dissolution of these structures. Interestingly, MuRF-1 deficient mice exhibit no major alterations in the levels of ubiquitination within the myocardium, suggesting redundant functionality among MuRFs in cardiac muscle [157].

2.5.2. MuRF-2. MuRF-2 anchors to the M-band via its association with p62 and NBR1 [23, 94]. Downregulation of MuRF-2 results in disrupted M-bands, in addition to perturbed intermediate filament and microtubule networks [169]. Consistent with the notion that MuRFs may have redundant functions in striated muscles, MuRF-2 deficient mice exhibit no apparent phenotype in the absence of physiological stress $[157,170]$. Interestingly though, homozygous MuRF-1/MuRF-2 double knock-out mice exhibit loss of Type II fibers in soleus muscle as well as severe cardiac and modest skeletal muscle hypertrophy due to increased protein synthesis, although proteasomal degradation is unaffected $[165,171]$. This suggests additional roles for MuRFs, possibly in transcriptional regulation and protein synthesis, which is consistent with their nuclear localization $[23,171]$.

2.5.3. MuRF-3. MuRF-3 localizes to the M-band by heterodimerizing with MuRF-1 or MuRF-2 [163]. Homozygous deletion of MuRF-3 in mice reveals its role in sarcomeric organization, as evidenced by increased sarcomeric length and upregulation of select proteins, including FHL-2 [172]. In spite of the increased sarcomeric length, MuRF-3 null mice do not exhibit cardiac hypertrophy in the absence of stress [172]. However, MuRF-3 null hearts are more prone to rupture following myocardial infarction [172]. Moreover, double knock-out mice of MuRF-1 and MuRF-3 exhibit skeletal myopathy and hypertrophic cardiomyopathy, as shown by accumulation of myosin at the subsarcolemma region, myofiber fragmentation, and reduced muscle contractility [158]. Interestingly, Z-disks, but not M-bands, are disrupted in the MuRF-1/MuRF-3 double knock-out mice [158]. Since MuRF-2 is present at M-bands, but not Z-disks, it is possible that it may compensate for the loss of MuRF-1 and MuRF3 with regards to protein turnover, thus contributing to the maintenance of M-band organization. This is consistent with MuRF proteins having redundant functions, at least partially, and highlights the importance of regulated proteasomal degradation in myofilament organization and contractility.

In addition to the MuRF family, another E3 ubiquitin ligase, cullin-3, is involved in the proteasomal degradation of small Ankyrin 1 (sAnk1), an integral membrane protein of the network sarcoplasmic reticulum that overlies M-bands and Z-disks [66]. However, since cullin-3 is localized at Zdisks rather than M-bands [66], it is highly possible that this process takes place at the former rather than the latter structure.

\section{Cardiac and Skeletal Myopathies Associated with M-Band Proteins}

A significant percentage of skeletal and cardiac myopathies are linked to genetic mutations in genes encoding sarcomeric, metabolic, and enzymatic proteins [173-176], many of which are localized to the M-region (Figure 2). Herein, we present a comprehensive overview of mutations associated with sarcomeric proteins of the M-region. In particular, we summarize early and current literature on genes encoding $\mathrm{M}$ region related proteins that are heavily mutated (referring the reader to focused reviews) (Tables 2 and 3 ) and emphasize the emerging roles of genes recently implicated in the development of skeletal and cardiac myopathies.

\subsection{Cytoskeletal Proteins and M-Band Myopathies}

3.1.1. Light Meromyosin. Hereditary myosin myopathies are a group of diseases caused by mutations in the heavy chain of myosin (MyHC) [259]. Mutations have been reported in the genes encoding three muscle-specific $\mathrm{MyHC}$ isoforms, including MYH7, which is expressed in slow-twitch skeletal and cardiac muscles, $M Y H 3$, which is abundantly expressed in embryonic skeletal muscles, and MYH6, which is selectively expressed in cardiac muscle [98]. Although multiple mutations have been identified along the entire length of MyHC [174, 214, 259], we only note those associated with the light meromyosin (LMM) portion of myosin that localizes to the M-band (Table 2). Over 86 disease-causing LMM mutations have been linked to the development of different skeletal and cardiac myopathies. Of those mutations, 78 map to MYH7 with 68 being missense mutations, 9 being insertions and/or deletions, and 1 being a nonsense mutation. Currently, the molecular alterations underlying the majority of these mutations are elusive.

3.1.2. Titin. Due to the development of next-generation sequencing, routine analysis of the giant titin gene (TTN) has been made possible. Consequently, TTN has emerged as a "hot spot" for inherited skeletal and cardiac myopathies affecting mankind [175]. Over 120 disease-causing titin mutations have been reported in patients with different skeletal and cardiac myopathies [175]. Of those mutations, 23 map 
TABLE 2: Disease-causing mutations in genes encoding structural proteins of the M-region.

\begin{tabular}{|c|c|c|c|c|c|}
\hline Protein & Mutation & Region on protein & Effect & Disease & Reference \\
\hline FHL-1 & K45SfsX1 & LIM domain 1 & Unknown & HCM & {$[177]$} \\
\hline FHL-1 & R95W & $\begin{array}{c}\text { Linker region between LIM } \\
\text { domain } 1 \text { and } 2\end{array}$ & Unknown & $\mathrm{RBM}$ & {$[178]$} \\
\hline FHL-1 & $\mathrm{C} 101 \mathrm{~F}$ & LIM domain 2 & Unknown & RBM & [179] \\
\hline FHL-1 & 102-104 del KFC & LIM domain 2 & Unknown & $\mathrm{RBM}$ & [179] \\
\hline FHL-1 & $\mathrm{C} 104 \mathrm{R} / \mathrm{Y}$ & LIM domain 2 & Unknown & $\mathrm{RBM}$ & {$[179,180]$} \\
\hline FHL-1 & $111-229$ del ins $G$ & LIM domain 2 & Unknown & EDMD & {$[181]$} \\
\hline FHL-1 & N112FfsX51 & LIM domain 2 & Unknown & EDMD & {$[181]$} \\
\hline FHL-1 & W122S/C & LIM domain 2 & Unknown & SPM & {$[182,183]$} \\
\hline FHL-1 & $\mathrm{H} 123 \mathrm{Y} / \mathrm{Q} / \mathrm{L} / \mathrm{R}$ & LIM domain 2 & Unknown & $\mathrm{RBM}$ & {$[178,184-186]$} \\
\hline FHL-1 & K124RfsX6 & LIM domain 2 & Unknown & EDMD & {$[181]$} \\
\hline FHL-1 & F127 ins $128 \mathrm{I}$ & LIM domain 2 & Unknown & XMPMA & [187] \\
\hline FHL-1 & $\mathrm{C} 132 \mathrm{~F}$ & LIM domain 2 & Unknown & RBM & {$[186]$} \\
\hline FHL-1 & $\mathrm{C} 150 \mathrm{Y} / \mathrm{R} / \mathrm{S}$ & LIM domain 2 & Unknown & RBM & {$[179,185,188]$} \\
\hline FHL-1 & 151-153 del VTC & LIM domain 2 & Unknown & RSS & [179] \\
\hline FHL-1 & $\mathrm{C} 153 \mathrm{Y} / \mathrm{R} / \mathrm{S} / \mathrm{W}$ & LIM domain 2 & Unknown & $\mathrm{RBM}$ & {$[186]$} \\
\hline FHL-1 & 153Stop & LIM domain 2 & Unknown & $\mathrm{HCM}$ & [177] \\
\hline FHL-1 & Delete exon 6 ins 84 bp & LIM domain 3 & $\begin{array}{l}\text { Loss of full length FHL-1A, } \\
\text { increase in FHL-1C }\end{array}$ & EDMD & [189] \\
\hline FHL-1 & K157VfsX36 & LIM domain 3 & Unknown & EDMD & {$[181]$} \\
\hline FHL-1 & A168GfsX195 & LIM domain 3 & Unknown & XMPMA & [187] \\
\hline FHL-1 & 194Stop & LIM domain 3 & $\begin{array}{l}\text { Premature stop codon and } \\
\text { truncated protein } \\
\text { corresponding to FHL-1C }\end{array}$ & XMPMA & {$[190]$} \\
\hline FHL-1 & 198Stop & LIM domain 3 & Unknown & $\mathrm{HCM}$ & {$[191]$} \\
\hline FHL-1 & F200fs $32 X$ & LIM domain 3 & Unknown & $\mathrm{HCM}$ & [192] \\
\hline FHL-1 & C209R & LIM domain 3 & Unknown & $\mathrm{EDMD} / \mathrm{HCM}$ & {$[181,193]$} \\
\hline FHL-1 & C224W & LIM domain 4 & Unknown & XMPMA & [187] \\
\hline FHL-1 & $\mathrm{H} 246 \mathrm{Y}$ & LIM domain 4 & Unknown & XMPMA & {$[190]$} \\
\hline FHL-1 & C273LfsX11 & LIM domain 4 & Unknown & EDMD & {$[181]$} \\
\hline FHL-1 & C276Y & LIM domain 4 & Unknown & EDMD & {$[181]$} \\
\hline FHL-1 & $\mathrm{C} 276 \mathrm{~S}$ & LIM domain 4 & Unknown & $\mathrm{HCM}$ & [177] \\
\hline FHL-1 & V280M & NLS of FHL-1B & Unknown & XMPMA & [190] \\
\hline FHL-1 & E281Stop & Extreme $\mathrm{COOH}$-terminus & Unknown & EDMD & {$[181]$} \\
\hline FHL-2 & G48S & LIM domain 1 & Loss of titin binding & DCM & [194] \\
\hline sMyBP-C & W236R & M-motif & $\begin{array}{l}\text { Loss of actin and myosin } \\
\text { binding }\end{array}$ & DA-1 & [195] \\
\hline sMyBP-C & R318Stop & $\operatorname{IgC} 2$ & $\begin{array}{l}\text { Premature stop codon and } \\
\text { truncated protein }\end{array}$ & LCCS4 & [196] \\
\hline sMyBP-C & Y856H & $\operatorname{IgC8}$ & Loss of myosin binding & DA-1 & [195] \\
\hline $\mathrm{MyH} 3$ & 841-841 del L & LMM & Reduced catalytic activity & $\begin{array}{l}\text { DA Sheldon-Hall } \\
\text { syndrome }\end{array}$ & [197] \\
\hline MyH 6 & $\begin{array}{l}\text { A1004S } \\
\text { E1457K }\end{array}$ & LMM & Unknown & $\mathrm{DCM}$ & {$[198]$} \\
\hline МyH 6 & Q1065H & LMM & Unknown & $\mathrm{HCM}$ & {$[198]$} \\
\hline МyH 6 & $\begin{array}{l}\text { R1116S } \\
\text { A1366D } \\
\text { A1443D } \\
\text { R1865Q }\end{array}$ & LMM & Unknown & $\mathrm{CHD}$ & {$[199]$} \\
\hline
\end{tabular}


TABLe 2: Continued.

\begin{tabular}{|c|c|c|c|c|c|}
\hline Protein & Mutation & Region on protein & Effect & Disease & Reference \\
\hline MyH 7 & 847-847 del K & LMM & Unknown & HCM & [200] \\
\hline MyH 7 & M852T & LMM & Unknown & $\mathrm{HCM}$ & {$[201]$} \\
\hline MyH 7 & $\mathrm{R} 858 \mathrm{C}$ & LMM & Unknown & $\mathrm{HCM}$ & {$[200]$} \\
\hline MyH 7 & R869G & LMM & Unknown & $\mathrm{HCM}$ & {$[201]$} \\
\hline MyH 7 & $\mathrm{R} 870 \mathrm{H}$ & LMM & Unknown & $\mathrm{HCM}$ & {$[202]$} \\
\hline MyH 7 & 883-883 del E & LMM & Unknown & $\mathrm{HCM}$ & {$[201]$} \\
\hline MyH 7 & E894G & LMM & Unknown & $\mathrm{HCM}$ & {$[200]$} \\
\hline MyH 7 & D906G & LMM & Unknown & HCM & [203] \\
\hline $\mathrm{MyH} 7$ & L908V & LMM & Unknown & HCM with CCD & {$[204]$} \\
\hline MyH 7 & E921K & LMM & Unknown & HCM & {$[200]$} \\
\hline MyH 7 & $\begin{array}{l}\text { E924K } \\
\text { E949K }\end{array}$ & LMM & Unknown & $\mathrm{HCM}$ & [205] \\
\hline MyH 7 & D928V & LMM & Unknown & $\mathrm{HCM}$ & [206] \\
\hline MyH 7 & E931K & LMM & Unknown & $\mathrm{HCM}$ & [200] \\
\hline MyH 7 & E935K & LMM & Unknown & $\mathrm{HCM}$ & [207] \\
\hline MyH 7 & $\mathrm{D} 953 \mathrm{H}$ & LMM & Unknown & $\mathrm{HCM}$ & [200] \\
\hline MyH 7 & T1019N & LMM & Unknown & DCM & [208] \\
\hline MyH 7 & R1053Q & LMM & Unknown & $\mathrm{HCM}$ & [209] \\
\hline MyH 7 & G1057S & LMM & Unknown & $\mathrm{HCM}$ & {$[200]$} \\
\hline $\mathrm{MyH} 7$ & L1135R & LMM & Unknown & $\mathrm{HCM}$ & {$[201]$} \\
\hline MyH 7 & R1193S & LMM & Unknown & $\mathrm{DCM}$ & {$[208]$} \\
\hline MyH 7 & E1218Q & LMM & Unknown & $\mathrm{HCM}$ & {$[201]$} \\
\hline MyH 7 & N1327K & LMM & $\begin{array}{l}\text { Reduced } \alpha \text {-helical content } \\
\text { of the rod domain }\end{array}$ & $\mathrm{HCM}$ & {$[210]$} \\
\hline MyH 7 & E1356K & LMM & $\begin{array}{l}\text { Reduced } \alpha \text {-helical content } \\
\text { of the rod domain }\end{array}$ & $\mathrm{HCM}$ & {$[211]$} \\
\hline MyH 7 & $\begin{array}{l}\text { E1377M } \\
\text { A1379T } \\
\text { R1382W }\end{array}$ & LMM & Unknown & $\mathrm{HCM}$ & {$[201]$} \\
\hline MyH 7 & R1420W & LMM & Unknown & $\mathrm{HCM}$ & {$[200]$} \\
\hline $\mathrm{MyH} 7$ & E1426K & LMM & Unknown & $\mathrm{DCM}$ & {$[208]$} \\
\hline MyH 7 & A1439P & LMM & Unknown & MPD1 & {$[212]$} \\
\hline MyH 7 & K1459N & LMM & Unknown & $\mathrm{HCM}$ & {$[200]$} \\
\hline $\mathrm{MyH} 7$ & L1467V & LMM & Unknown & Congenital myopathy & {$[213]$} \\
\hline MyH 7 & L1481P & LMM & Unknown & MPD1 & {$[214]$} \\
\hline MyH 7 & R1500W & LMM & $\begin{array}{l}\text { Reduced } \alpha \text {-helical content } \\
\text { of the rod domain }\end{array}$ & $\mathrm{DCM}$ & {$[215]$} \\
\hline MyH 7 & $\begin{array}{c}\text { R1500P } \\
1617-1617 \text { del K }\end{array}$ & LMM & Unknown & Laing distal myopathy & {$[216]$} \\
\hline MyH 7 & 1508-1508 del E & LMM & Unknown & MPD1 & {$[217]$} \\
\hline MyH 7 & T1513S & LMM & Unknown & $\mathrm{HCM}$ & [200] \\
\hline MyH 7 & Q1541P & LMM & Unknown & MPD1 & {$[214]$} \\
\hline MyH 7 & E1555K & LMM & $\begin{array}{l}\text { Reduced } \alpha \text {-helical content } \\
\text { of the rod domain }\end{array}$ & $\mathrm{HCM}$ & {$[218]$} \\
\hline MyH 7 & $\mathrm{R} 1588 \mathrm{P}$ & LMM & Unknown & MPD1 & {$[213]$} \\
\hline MyH 7 & L1591P & LMM & Unknown & MPD1 & {$[219]$} \\
\hline MyH 7 & L1597R & LMM & Unknown & $\begin{array}{l}\text { Axial myopathy, } \\
\text { contractual myopathy }\end{array}$ & {$[220]$} \\
\hline MyH 7 & T1599P & LMM & Unknown & MPD1 & {$[214]$} \\
\hline MyH 7 & A1603P & LMM & Unknown & MPD1 & {$[217]$} \\
\hline
\end{tabular}


TABle 2: Continued.

\begin{tabular}{|c|c|c|c|c|c|}
\hline Protein & Mutation & Region on protein & Effect & Disease & Reference \\
\hline MyH 7 & R1608P & LMM & Unknown & $\begin{array}{c}\text { Congenital myopathy, } \\
\text { HCM }\end{array}$ & {$[214]$} \\
\hline MyH 7 & L1612P & LMM & Unknown & MPD1 & [214] \\
\hline MyH 7 & 1617-1617 del K & LMM & Unknown & MPD1, DCM & {$[214,216]$} \\
\hline MyH 7 & $\mathrm{R} 1634 \mathrm{~S}$ & LMM & Unknown & DCM & [208] \\
\hline MyH 7 & $\begin{array}{l}\text { A1636P } \\
\text { L1646P } \\
\text { R1662P }\end{array}$ & LMM & Unknown & MPD1 & {$[214]$} \\
\hline MyH 7 & A1663P & LMM & Unknown & MPD1 & {$[216]$} \\
\hline MyH 7 & 1669-1669 del E & LMM & Unknown & MPD1 & {$[214]$} \\
\hline MyH 7 & V1691M & LMM & Unknown & $\mathrm{HCM}$ & {$[201]$} \\
\hline MyH 7 & L1706P & LMM & Unknown & MPD1 & [216] \\
\hline MyH 7 & R1712W & LMM & Unknown & $\mathrm{HCM}$ & [210] \\
\hline MyH 7 & L1723P & LMM & Unknown & CCD & {$[221]$} \\
\hline MyH 7 & 1729-1729 del K & LMM & Unknown & Laing distal myopathy & {$[216]$} \\
\hline $\mathrm{MyH} 7$ & E1753K & LMM & Unknown & $\mathrm{HCM}$ & {$[210]$} \\
\hline MyH 7 & A1766T & LMM & Unknown & LVNC & {$[222]$} \\
\hline MyH 7 & E1768K & LMM & $\begin{array}{l}\text { Increased } \alpha \text {-helical content } \\
\text { of the rod domain }\end{array}$ & $\mathrm{HCM}$ & {$[200]$} \\
\hline MyH 7 & S1776G & LMM & Unknown & $\mathrm{HCM}$ & {$[223]$} \\
\hline MyH 7 & A1777T & LMM & Unknown & HCM & [201] \\
\hline MyH 7 & 1784-1784 del K & LMM & Unknown & MPD1, MSM & {$[219,224]$} \\
\hline MyH 7 & L1793P & LMM & $\begin{array}{l}\text { Destabilization of the thick } \\
\text { filaments }\end{array}$ & HCM with MSD & {$[225,226]$} \\
\hline MyH 7 & 1793-1793 del L & LMM & Unknown & MPD1 & {$[214]$} \\
\hline MyH 7 & $\mathrm{E} 1801 \mathrm{~K}$ & LMM & Unknown & MPD1, DCM, HCM & {$[214,217]$} \\
\hline MyH 7 & $\mathrm{~T} 1834 \mathrm{M}$ & LMM & Unknown & $\mathrm{HCM}$ & [200] \\
\hline MyH 7 & R1845W & LMM & $\begin{array}{l}\text { Alters interactions between } \\
\text { filaments }\end{array}$ & MSM & {$[227]$} \\
\hline MyH 7 & E1856K & LMM & Unknown & $\begin{array}{c}\text { Late onset myopathy } \\
\text { with cardiac } \\
\text { involvement }\end{array}$ & {$[228]$} \\
\hline MyH 7 & E1883K & LMM & $\begin{array}{l}\text { Destabilization of the thick } \\
\text { filaments }\end{array}$ & $\mathrm{HCM}$ & {$[226,229]$} \\
\hline MyH 7 & H1901L & LMM & $\begin{array}{l}\text { Alters interactions between } \\
\text { filaments }\end{array}$ & MSM & {$[230]$} \\
\hline MyH 7 & E1914K & LMM & Unknown & DCM & {$[214]$} \\
\hline MyH 7 & N1918K & LMM & Unknown & LVNC & {$[231]$} \\
\hline MyH 7 & T1929M & LMM & Unknown & $\mathrm{HCM}$ & {$[200]$} \\
\hline $\mathrm{MyH} 7$ & Stop1936W & LMM & Unknown & MSM & {$[232]$} \\
\hline Myomesin & $\begin{array}{l}\text { Aberrant splicing of exon } \\
17 \mathrm{a}\end{array}$ & EH-motif & $\begin{array}{l}\text { Premature stop codon and } \\
\text { truncated protein }\end{array}$ & MD1 & {$[233]$} \\
\hline Myomesin & V1490I & $\operatorname{Ig} 12$ & Reduced dimerization & $\mathrm{HCM}$ & {$[234]$} \\
\hline Obscurin & R4344Q & $\operatorname{Ig} 58$ & Loss of titin binding & HCM & {$[235]$} \\
\hline Titin & S33705LfsX4 & $\mathrm{TK}$ & Unknown & LGMD2J & {$[236]$} \\
\hline Titin & N34020TfsX9 & $\mathrm{TK}$ & $\begin{array}{c}\text { Increased structural } \\
\text { stability of TK, loss of } \\
\text { interactions with proteins } \\
\text { partners of TK }\end{array}$ & MmD-HD & {$[175]$} \\
\hline Titin & R34091W & TK & Unknown & HMERF & {$[23]$} \\
\hline Titin & R34175Stop & MIg1 & Unknown & $\mathrm{MmD}-\mathrm{HD}$ & {$[175]$} \\
\hline
\end{tabular}


TABLE 2: Continued.

\begin{tabular}{|c|c|c|c|c|c|}
\hline Protein & Mutation & Region on protein & Effect & Disease & Reference \\
\hline Titin & 32664-32665 del ins K & MIg2 & Unknown & $\mathrm{HCM}$ & {$[237]$} \\
\hline Titin & P34617QinsX3 & MIs2 & Unknown & $\mathrm{CNM}$ & {$[238]$} \\
\hline Titin & R34637Q & MIg4 & Unknown & DCM & [239] \\
\hline Titin & A32606fsX7 & MIg5 & Unknown & DCM & [240] \\
\hline Titin & Q35176HfsX9 & MIg5 & Truncated titin & $\begin{array}{l}\text { MmD-HD } \\
\text { (EOMFC) }\end{array}$ & {$[241]$} \\
\hline Titin & Q35278Stop & MIs4 & Unknown & MmD-HD & {$[175]$} \\
\hline Titin & G35340VfsX65 & MIg6 & Unknown & CNM & {$[238]$} \\
\hline Titin & 33710-33711 del ins $\mathrm{K}$ & MIg6 & Unknown & HCM & {$[237]$} \\
\hline Titin & S35469SfsX11 & MIg7 & Unknown & $\mathrm{MmD}-\mathrm{HD}$ & {$[175]$} \\
\hline Titin & K35524RfsX22 & MIs6 & Unknown & $\begin{array}{l}\text { MmD-HD } \\
\text { (EOMFC) }\end{array}$ & {$[241]$} \\
\hline Titin & 32986-32987 del ins K & MIg8 & Unknown & DCM & {$[240]$} \\
\hline Titin & M35859T & MIs7 & Unknown & ARVC & {$[242]$} \\
\hline Titin & S35883QfsX10 & MIs7 & Unknown & TMD & {$[243]$} \\
\hline Titin & $\begin{array}{l}\text { Q35927-35931W del ins } \\
\text { VKQK }\end{array}$ & MIg10 & Truncated titin & $\begin{array}{l}\text { TMD, LGMD2J, } \\
\text { MD }\end{array}$ & {$[236,244,245]$} \\
\hline Titin & $\mathrm{H} 35946 \mathrm{P}$ & MIg10 & Unknown & TMD & [246] \\
\hline Titin & $\mathrm{I} 35947 \mathrm{~N}$ & MIg10 & Unknown & TMD & {$[247]$} \\
\hline Titin & L35956P & MIg10 & Unknown & TMD & [244] \\
\hline Titin & K35963NfsX9 & MIg10 & Unknown & TMD, CNM & {$[238,243]$} \\
\hline Titin & Q35964Stop & MIg10 & Truncated titin & TMD & [243] \\
\hline
\end{tabular}

Note: nomenclature refers to the canonical full-length human isoforms; FHL-1, NP_001153174.1, sMyBP-C, AAI43503.1, MyH 3, NP_002461.2, MyH 6, NP_002462.2, MyH 7, NP_000248.2, myomesin, CAF18565.1, obscurin, CAC44768.1, titin, NP_001254479.2. HCM: hypertrophic cardiomyopathy, RBM: reducing body myopathy, XMPMA: X-linked myopathy with postural muscle atrophy, SPM: scapuloperoneal myopathy, RSS: rigid spine syndrome, EDMD: Emery-Dreifuss muscular dystrophy, DCM: dilated cardiomyopathy, DA-1: distal arthrogryposis type 1, LCCS4: lethal congenital contracture syndrome type 4, MPD1: Laing distal myopathy, CHD: congenital heart defect, CCD: central core disease, MSM: myosin storage myopathy, LVNC: left ventricular noncompaction, MD1: myotonic dystrophy type 1, LGMD2J: limb-girdle muscular dystrophy type 2J, MmD-HD: multiminicore disease with heart disease, HMERF: hereditary myopathy with early respiratory failure, CNM: centronuclear myopathy, EOMFC: early-onset myopathy with fatal cardiomyopathy, ARVC: arrhythmogenic right ventricular cardiomyopathy, TMD: tibial muscular dystrophy, MD: muscle disease, NLS: nuclear localization sequence, TK: titin kinase, MIgX: titin M-band IgX, MyH: myosin heavy chain, and LMM: light meromyosin.

to the M-band region of titin, a significant percentage given the relatively small size of titin's M-band region $(\sim 200 \mathrm{kDa})$ compared to the rest of the molecule ( 2-3 MDa). Of these 23 mutations, 9 are frameshift, 3 are nonsense, 6 are missense, and 5 are insertions and/or deletions (Table 2). While the mutations associated with the development of cardiomyopathies affect several domains throughout the Mband portion of titin, the ones that are linked to skeletal myopathies are mainly contained within the last Ig domain of titin, MIg10 [260].

3.1.3. Obscurin. Mutations in OBSCN, the gene encoding giant obscurins, have been linked to the development of hypertrophic cardiomyopathy (HCM) [13, 261]. Specifically, the presence of a missense mutation results in an R4344Q substitution within the Ig58 domain of obscurin [235]. In vitro studies have shown that the R4344Q mutation results in decreased binding of obscurin Ig58/Ig59 domains to the titin Ig9/Ig10 domains, which localize at the Z/I junction. However, the pathological effects of this mutation on sarcomeric assembly or $\mathrm{Ca}^{2+}$ homeostasis are still unknown.
3.1.4. Myosin Binding Protein-C Slow. MYBPC1 encodes the slow isoform of MyBP-C and has been directly implicated in the development of severe and lethal skeletal myopathies $[10,195,196]$. Two autosomal dominant missense mutations have been identified to date, W236R and $\mathrm{Y} 856 \mathrm{H}$, which are linked to the development of distal arthrogryposis type-1 (DA1), a severe skeletal myopathy selectively affecting distal muscles [195]. Recent work from our group has demonstrated that the W236R and $\mathrm{Y} 856 \mathrm{H}$ mutations abolish the ability of the $\mathrm{NH}_{2}$ and $\mathrm{COOH}$ termini, respectively, to bind native actin and/or myosin and to regulate the formation of actomyosin cross-bridges in vitro [262]. Moreover, MYBC1 has been causally associated with the development of lethal congenital contractual syndrome type-4 (LCCS4), a neonatal lethal form of arthrogryposis myopathy [196] that most likely results in a null phenotype. A homozygous nonsense mutation in the C2 domain of sMyBP-C (R318Stop) consists of the molecular basis of LCCS4. Given that all three mutations are encoded by exons that are constitutively expressed, they are present in all sMyBP-C variants, including those that carry a unique $\mathrm{COOH}$-terminal insertion and preferentially localize to the periphery of the M-band [10, 263]. 
Table 3: Proteins with enzymatic activity at the M-region and related diseases.

\begin{tabular}{|c|c|c|c|c|}
\hline Protein & Mutation & Effect & Disease & Reference \\
\hline$\beta$-Enolase & $\begin{array}{l}\text { G156N } \\
\text { G374E }\end{array}$ & Unknown & GSD XIII & [248] \\
\hline MuRF1 & S5L & Unknown & $\mathrm{HCM}$ & [249] \\
\hline MuRF1 & F73S & Unknown & $\mathrm{HCM}$ & [249] \\
\hline MuRF1 & $\mathrm{R} 86 \mathrm{C} / \mathrm{H}$ & Unknown & HCM & [249] \\
\hline MuRF1 & $\mathrm{I} 101 \mathrm{~F}$ & Unknown & $\mathrm{HCM}$ & [249] \\
\hline MuRF1 & $\mathrm{T} 232 \mathrm{M}$ & Unknown & $\mathrm{HCM}$ & [249] \\
\hline MuRF1 & E299Stop & Unknown & $\mathrm{HCM}$ & [249] \\
\hline MuRF1 & M305I & Unknown & $\mathrm{HCM}$ & [249] \\
\hline MuRF1 & $\mathrm{A} 318 \mathrm{D}$ & Unknown & $\mathrm{HCM}$ & [249] \\
\hline MuRF2 & $\mathrm{C} 50 \mathrm{Y}$ & Unknown & $\mathrm{HCM}$ & [249] \\
\hline MuRF2 & P79A & Unknown & $\mathrm{HCM}$ & [249] \\
\hline MuRF2 & Q187fs & Unknown & HCM & [249] \\
\hline MuRF2 & L241M & Unknown & $\mathrm{HCM}$ & [249] \\
\hline MuRF2 & $\mathrm{S} 252 \mathrm{~F}$ & Unknown & $\mathrm{HCM}$ & [249] \\
\hline MuRF2 & E371fs & Unknown & $\mathrm{HCM}$ & [249] \\
\hline MuRF2 & P392T & Unknown & HCM & [249] \\
\hline MuRF2 & K425N & Unknown & HCM & [249] \\
\hline MuRF2 & $\mathrm{A} 488 \mathrm{~T}$ & Unknown & $\mathrm{HCM}$ & [249] \\
\hline MuRF2 & T506S & Unknown & $\mathrm{HCM}$ & [249] \\
\hline MuRF2 & H523W & Unknown & $\mathrm{HCM}$ & [249] \\
\hline MuRF2 & F538fs & Unknown & $\mathrm{HCM}$ & [249] \\
\hline MuRF3 & P9L & Unknown & $\mathrm{HCM}$ & [249] \\
\hline MuRF3 & G94C & Unknown & $\mathrm{HCM}$ & [249] \\
\hline MuRF3 & P115S & Unknown & $\mathrm{HCM}$ & [249] \\
\hline MuRF3 & L163P & Unknown & $\mathrm{HCM}$ & [249] \\
\hline MuRF3 & A221V & Unknown & $\mathrm{HCM}$ & [249] \\
\hline MuRF3 & R249Q & Unknown & $\mathrm{HCM}$ & [249] \\
\hline MuRF3 & $\mathrm{R} 269 \mathrm{H}$ & Unknown & $\mathrm{HCM}$ & [249] \\
\hline MuRF3 & $\mathrm{K} 270 \mathrm{~N}$ & Unknown & $\mathrm{HCM}$ & [249] \\
\hline MuRF3 & P346T & Unknown & $\mathrm{HCM}$ & [249] \\
\hline MuRF3 & G373D & Unknown & HCM & [249] \\
\hline PFK & R39P/L & Unknown & GSD VII & {$[250,251]$} \\
\hline PFK & G57V & Unknown & GSD VII & [252] \\
\hline PFK & G80fs $4 \mathrm{X}$ & Unknown & GSD VII & [252] \\
\hline PFK & R95Stop & Unknown & GSD VII & [253] \\
\hline PFK & R100Q & Unknown & GSD VII & [254] \\
\hline PFK & S108C & Unknown & GSD VII & [252] \\
\hline PFK & G209D & Unknown & GSD VII & {$[254]$} \\
\hline PFK & N309G & Unknown & GSD VII & {$[255]$} \\
\hline PFK & D543A & Unknown & GSD VII & {$[250]$} \\
\hline PFK & D591A & Unknown & GSD VII & [252] \\
\hline PFK & P668Q & Unknown & GSD VII & [251] \\
\hline PFK & W686C & Unknown & GSD VII & [254] \\
\hline PFK & $\mathrm{R} 696 \mathrm{H}$ & Unknown & GSD VII & {$[251]$} \\
\hline PFK & 78 bp del exon 5 & Unknown & GSD VII & {$[254]$} \\
\hline PFK & 5 or 12 bp del exon 7 & Unknown & GSD VII & {$[250]$} \\
\hline
\end{tabular}


TABLE 3: Continued.

\begin{tabular}{lcclc}
\hline Protein & Mutation & Effect & Disease & Reference \\
\hline PFK & 75 bp del exon 15 & Unknown & GSD VII & [256] \\
PFK & $\begin{array}{c}\text { Retention of intron 13, } \\
\text { truncated protein } \\
\text { Retention of intron }\end{array}$ & Unknown & GSD VII & \\
PFK & $\begin{array}{l}\text { 16, truncated protein } \\
169 \text { bp del exon } 19\end{array}$ & Unknown & GSD VII \\
PFK & Unknown & GSD VII & [257] \\
\hline
\end{tabular}

Note: nomenclature refers to the canonical full-length human isoforms; beta-enolase, NP_001967.3, MuRF1, NP_115977.2, MuRF2, Q9BYV6.2, MuRF3, NP_912730.2, PFK, and NP_000280.1. HCM: hypertrophic cardiomyopathy; GSD: glycogen storage disease.

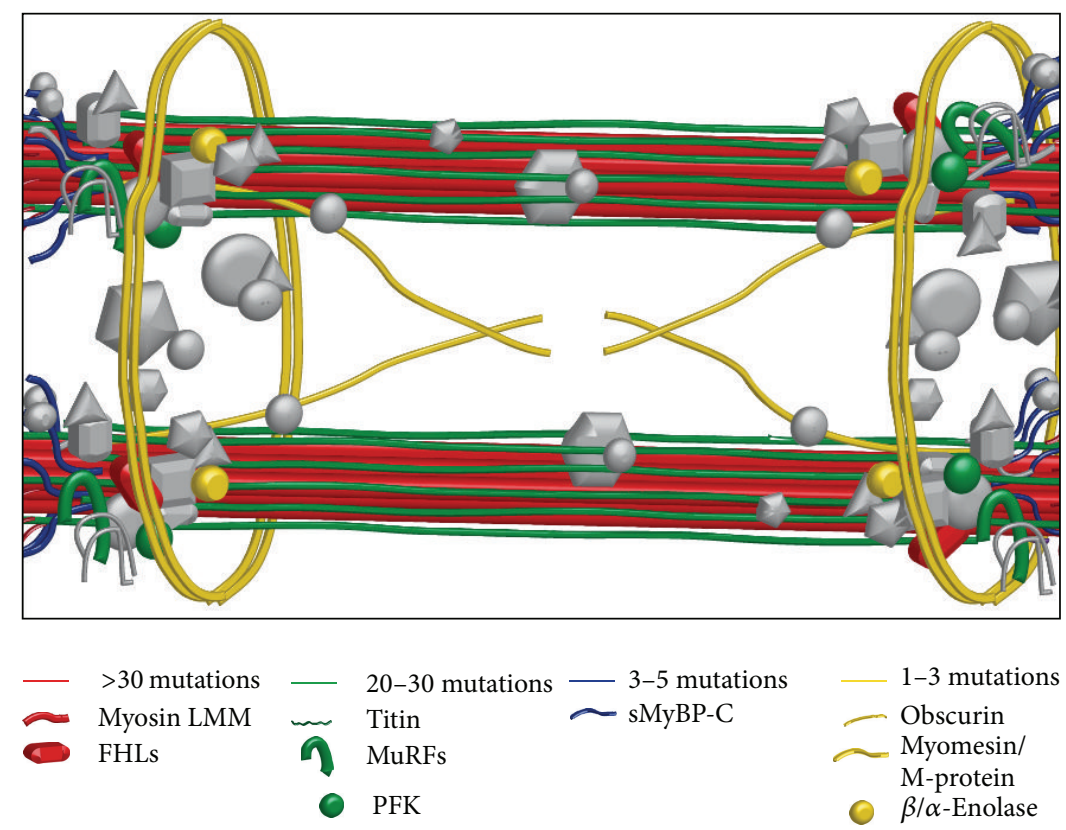

Figure 2: M-region proteins associated with skeletal and cardiac myopathies. Schematic representation of sarcomeric M-region proteins linked to the development of skeletal and cardiac myopathies. Proteins exhibiting no known disease-linked mutations are shown in grey color.

3.1.5. Myomesin. The gene that encodes myomesin, MYOM1, has been directly linked to the development of HCM [234]. Specifically, a missense mutation resulting in V1490I substitution within the $\mathrm{COOH}$-terminal Ig12 domain reduces the ability of myomesin to homodimerize (Table 2). Although the molecular etiology of HCM due to the V1490I substitution is still unknown, it is likely that mutant myomesin fails to crosslink myosin thick filaments at the M-band [234].

In addition, myomesin has been associated with myotonic dystrophy type 1 (DM1), a multisystem disease characterized by myotonia, muscle weakness, cardiac conduction defects, insulin resistance, and mental retardation. DM1 is caused by expansion of a CTG repeat present in the $3^{\prime}$ UTR region of dystrophia myotonica-protein kinase, DMPK [264], resulting in aggregation of muscle blind-like (MBNL) protein. MBNL is an RNA binding protein that regulates the alternative splicing of the MYOM1 gene [233]. Specifically, exon 17a encoding the EH-motif that links the third and fourth FnIII domains of myomesin is developmentally regulated. In normalcy, exon 17a is expressed in embryonic heart, while in heart failure it is also expressed in adult myocardium, as a compensatory mechanism to render the heart muscle more compliant $[265,266]$. In the case of DM1 patients, loss of MBNL function leads to inclusion of exon 17a in adult cardiac muscle resulting in expression of a myomesin form that compromises the ability of the afflicted muscles to withstand stress and generate force [233].

3.1.6. Four and a Half LIM (FHL) Domain Proteins. FHL1 and FHL-2 have been directly linked to the development of skeletal and cardiac myopathies [176, 267]. Mutations in the FHL1 gene, encoding FHL-1, have been linked to the development of five distinct skeletal muscle diseases, including reducing body myopathy (RBM), X-linked myopathy with postural muscle atrophy (XMPMA), scapuloperoneal myopathy (SPM), rigid spine syndrome (RSS), and EmeryDreifuss muscular dystrophy (EDMD) [176]. Since a number of thorough reviews on FHL-1 associated myopathies have 
been published prior to 2011 [176, 184, 267], we will focus our discussion on new information, originating after 2011. For consistency purposes, a complete listing of all known FHL-1 mutations to date is listed in Table 2.

Since 2011, six additional mutations in FHL-1 have been linked to the development of RBM and EDMD, increasing the total number of FHL-1 skeletal myopathy linked mutations from 29 prior to 2011 to 35 after 2011. Specifically, RBM linked mutation $\mathrm{R} 95 \mathrm{~W}$ is located within the linker region between LIM domains 1 and 2, while C104Y, H123R, C126Y, and C153S/W substitutions are present in LIM domain 2 $[178,180,268]$. Moreover, deletion of exon 6 resulting in loss of full length FHL-1 was linked to the development of EDMD [189]. Recently, Binder et al. demonstrated that patients, who presented with XMPMA due to mutations in the FHL1 gene, also suffer from reduced cardiac function [269]. This was observed in hemizygote males as well as heterozygote females carrying one of the following mutations, C224W, H246Y, V280M, and A168fsX195 [269].

Furthermore, six novel mutations in FHL-1 have been directly linked with the development of HCM, by generating truncated or deleterious FHL-1 proteins. These include 2 frameshift mutations at residues 45 and 200 within LIM domains 1 and 3, respectively, 2 nonsense mutations generating premature termination codons at residues 153 and 198 within LIM domains 2 and 3, respectively, and 2 missense mutations, C209R and C276S, both located within LIM domain $3[177,191,192]$. In addition to the expression of truncated or poisonous forms of FHL-1, the levels of FHL1 are also altered in cardiomyopathies. In particular, patients with HCM exhibit 2-fold increased expression of FHL1, with a subsequent increase in protein levels of FHL-1 [270], while patients diagnosed with end-stage dilated cardiomyopathy (DCM) show a 3.5-fold decrease in the levels of FHL-1 transcripts resulting in reduced protein expression [271].

Although mutations in FHL-1 protein are commonly linked to the development of skeletal and cardiac myopathies, we are just beginning to unravel the molecular mechanisms underlying the pathogenesis of these myopathies. Recently, Wilding et al. showed that select RBM (H123Y, C132F, and C153Y), SPM (W122S), and XMPMA (F127 ins 128I and C224W) FHL-1 mutants accumulate in reducing bodies or protein aggregates when overexpressed in $\mathrm{C} 2 \mathrm{C} 12$ cells [272]. These reducing bodies are phenotypically similar to those found in patients suffering from the corresponding diseases. In addition, these same mutations result in impaired myoblast differentiation when overexpressed in $\mathrm{C} 2 \mathrm{C} 12$ cells, consistent with the loss of normal FHL-1 function [272]. Conversely, select EDMD (K157VfsX36, C273LfsX11, C276Y, and E281Stop) and EDMD/HCM (C209R) FHL-1 mutations result in reduced protein expression when overexpressed in $\mathrm{C} 2 \mathrm{C} 12$ cells, suggesting impaired transcriptional regulation and/or protein stability and degradation [272]. Thus, these studies are the first to suggest potential molecular alterations that underlie the different FHL-1 linked myopathies.

FHL-2 has also been associated with heart failure progression and the development of DCM. A missense mutation, G48S, in the first LIM domain of FHL-2 has been identified in a patient with familial DCM [194] (Table 2). The presence of this mutation reduces the ability of mutant FHL-2 protein to bind titin, suggesting a structural role for FHL-2 at the Mband.

3.2. Metabolic Enzymes and M-Band Myopathies. In addition to alterations in genes encoding cytoskeletal proteins, mutations in the metabolic enzymes PFK, $\beta$-enolase, and AMPD that localize at the M-band have been linked to the development of skeletal and cardiac myopathies; this group of diseases is collectively classified as "metabolic myopathies." Affected individuals present with muscle weakness, occasionally triggered by exercise, chronic respiratory failure, muscle rigidity, and decreased voluntary contractions [273]. Tarui disease, also referred to as glycogen storage disease (GSD) type VII, is a rare disorder involving impaired glycogen metabolism due to PFK deficiency and is characterized by exercise intolerance, myalgias, muscle cramps, and episodic myoglobinuria [252]. To date, 21 mutations in the PFK gene have been linked to the development of Tarui/GSD type VII disease (Table 3) [252, 255] (for a thorough discussion on Tarui disease and the molecular details of the identified mutations, please see [252]). Another form of GSD, referred to as GSD type XIII, is linked to defects in $\beta$-enolase. Two missense mutations (G156N and G374E) in the $\beta$-enolase gene have been linked to the development of GSD type XIII. These mutations result in decreased protein levels leading to a dramatic reduction ( $~ 95 \%)$ in cellular enolase activity [248] (Table 3).

Lastly, missense and truncation mutations in the human $A M P D 1$ gene have been linked with AMPD deficiency in skeletal muscle [274-277]. Specifically, the Q12X nonsense mutation gives rise to a premature stop codon leading to the generation of truncated $\mathrm{mRNA}$ and loss of AMPD-1 protein [275]. In addition, the P48L, Q156H, K287I, R388W, and $\mathrm{R} 425 \mathrm{H}$ missense mutations result in expression of mutant AMPD-1 proteins with negligible enzymatic activity [274, 276, 277], while a deletion mutation (IVS2 del CTTT) leads to expression of multiple inactive spliced forms of the protein [277]. While loss of AMPD-1 may be partially compensated by isoforms encoded by AMPD3 [278], the importance of AMPD-1 in maintaining optimal AMP levels is underscored by the severe effects of many of these disease-linked mutations.

\subsection{Ubiquitin Ligases and M-Band Myopathies}

3.3.1. MuRFs. It is only very recently that mutations in members of the MuRF family have been linked to the development of hypertrophic cardiomyopathies. Su et al. recently identified 8, 12, and 10 mutations in MuRF-1, MuRF-2, and MuRF3 genes, respectively (Table 3) [249]. Interestingly, these mutations are suggested to modify the severity of the HCM phenotype but not cause it per se. Moreover, select HCM linked MuRF-1 and MuRF-2 mutations cosegregate with mutations in genes encoding sarcomeric proteins, that is, MYH7, MYBPC3, MYC2, and MYL3. However, the contribution of these MuRF-1 and MuRF-2 mutations in the pathogenesis of HCM is still unknown. 
In addition, the expression profile of MuRF-1 is differentially regulated in response to pathophysiological processes, such as aging, atrophy, and senescence. Specifically, the levels of MuRF-1 are significantly increased in the initial phases of muscle disuse and atrophy in humans; this is consistent with a decrease in muscle fiber size [279]. Conversely, in aged skeletal muscle, the levels of MuRF-1 are decreased, coinciding with slowing of muscle atrophy [279]. Moreover, muscle loss is often associated with chronic diseases, such as cirrhosis and heart failure. Muscle biopsies obtained from malnourished cirrhotic patients exhibiting muscle atrophy contain increased amounts of MuRF-1 [280]. Similarly, MuRF-1's expression is upregulated in skeletal muscles of patients with chronic heart failure [281]. However, this trend is reversed in the same patients following exercise training [281].

Although the molecular etiologies underlying the differential expression of the MuRF proteins in skeletal and cardiac myopathies are yet to be defined, it is apparent that they play key roles in regulating muscle fiber size and muscle loss [282].

\section{Perspectives and Future Directions}

Throughout the last decade, it has become clear that in addition to its structural role, the M-region also acts as a mechanosensor, signal transduction center, and metabolic hub (Figure 1 and Table 1). Distinct mutations or alterations in the expression levels of M-band proteins have been implicated in the development of skeletal and cardiac muscle diseases. To date, more than 210 distinct mutations affect proteins that localize to the M-region. Notably, a striking 163 missense and nonsense mutations, 21 frameshift mutations, and 26 deletions/insertions within genes encoding M-region related proteins (Figure 2 and Tables 2 and 3) have been associated with the development of different forms of skeletal and cardiac myopathies. The severity of these diseases can vary dramatically, depending on the nature of the mutation and the role of the affected protein. Our current understanding of the molecular pathophysiology of individual mutations is still incomplete and only just emerging in most cases. Deciphering how these mutations alter M-band structure, contractile activity, signaling networks, posttranslational modifications, and energy production will aid in improving clinical diagnosis and developing individualized therapeutic approaches for affected individuals.

\section{Conflict of Interests}

The authors declare that there is no conflict of interests regarding the publication of this paper.

\section{Acknowledgments}

This work was supported by grants to Li-Yen $\mathrm{R}$. Hu (NIH, 5 T32AR7592), Maegen A. Ackermann (NIH, K99 KHL116778A), and Aikaterini Kontrogianni-Konstantopoulos (AHA, 14GRNT20380360).

\section{References}

[1] H. M. Eppenberger and J.-C. Perriard, Developmental Processes in Normal and Diseased Muscle, S. Karger AG, Basel, Switzerland, 1984.

[2] M. Sjöström and J. M. Squire, "Cryo-ultramicrotomy and myofibrillar fine structure: a review," Journal of Microscopy, vol. 111, no. 3, pp. 239-278, 1977.

[3] H. A. AL-Khayat, R. W. Kensler, E. P. Morris, and J. M. Squire, "Three-dimensional structure of the M-region (bare zone) of vertebrate striated muscle myosin filaments by single-particle analysis," Journal of Molecular Biology, vol. 403, no. 5, pp. 763776, 2010.

[4] P. Luther and J. Squire, "Three dimensional structure of the vertebrate muscle M region," Journal of Molecular Biology, vol. 125, no. 3, pp. 313-324, 1978.

[5] I. Agarkova, E. Ehler, S. Lange, R. Schoenauer, and J.-C. Perriard, "M-band: a safeguard for sarcomere stability?" Journal of Muscle Research and Cell Motility, vol. 24, no. 2-3, pp. 191-203, 2003.

[6] W. M. J. Obermann, M. Gautel, F. Steiner, P. F. M. van der Ven, K. Weber, and D. O. Fürst, "The structure of the sarcomeric $\mathrm{M}$ band: localization of defined domains of myomesin, Mprotein, and the $250-\mathrm{kD}$ carboxy-terminal region of titin by immunoelectron microscopy," The Journal of Cell Biology, vol. 134, no. 6, pp. 1441-1453, 1996.

[7] P. A. Kenny, E. M. Liston, and D. G. Higgins, "Molecular evolution of immunoglobulin and fibronectin domains in titin and related muscle proteins," Gene, vol. 232, no. 1, pp. 11-23, 1999.

[8] R. Schoenauer, S. Lange, A. Hirschy, E. Ehler, J.-C. Perriard, and I. Agarkova, "Myomesin 3, a novel structural component of the M-band in striated muscle," Journal of Molecular Biology, vol. 376, no. 2, pp. 338-351, 2008.

[9] A. Kontrogianni-Konstantopoulos, E. M. Jones, D. B. van Rossum, and R. J. Bloch, "Obscurin is a ligand for small ankyrin 1 in skeletal muscle," Molecular Biology of the Cell, vol. 14, no. 3, pp. 1138-1148, 2003.

[10] M. A. Ackermann, L.-Y. R. Hu, A. L. Bowman, R. J. Bloch, and A. Kontrogianni-Konstantopoulos, "Obscurin interacts with a novel isoform of MyBP-C slow at the periphery of the sarcomeric M-band and regulates thick filament assembly," Molecular Biology of the Cell, vol. 20, no. 12, pp. 2963-2978, 2009.

[11] P. Bagnato, V. Barone, E. Giacomello, D. Rossi, and V. Sorrentino, "Binding of an ankyrin-1 isoform to obscurin suggests a molecular link between the sarcoplasmic reticulum and myofibrils in striated muscles," Journal of Cell Biology, vol. 160, no. 2, pp. 245-253, 2003.

[12] M. J. Flick and S. F. Konieczny, "The muscle regulatory and structural protein MLP is a cytoskeletal binding partner of betaI-spectrin," Journal of Cell Science, vol. 113, part 9, pp. 15531564,2000

[13] A. Kontrogianni-Konstantopoulos, M. A. Ackermann, A. L. Bowman, S. V. Yap, and R. J. Bloch, "Muscle giants: molecular scaffolds in sarcomerogenesis," Physiological Reviews, vol. 89, no. 4, pp. 1217-1267, 2009.

[14] I. Agarkova and J.-C. Perriard, "The M-band: an elastic web that crosslinks thick filaments in the center of the sarcomere," Trends in Cell Biology, vol. 15, no. 9, pp. 477-485, 2005.

[15] D. O. Fürst, M. Osborn, R. Nave, and K. Weber, "The organization of titin filaments in the half-sarcomere revealed by monoclonal antibodies in immunoelectron microscopy: a map of ten nonrepetitive epitopes starting at the $\mathrm{Z}$ line extends close 
to the M line," The Journal of Cell Biology, vol. 106, no. 5, pp. 1563-1572, 1988.

[16] M. Gautel, K. Leonard, and S. Labeit, "Phosphorylation of KSP motifs in the C-terminal region of titin in differentiating myoblasts," The EMBO Journal, vol. 12, no. 10, pp. 3827-3834, 1993.

[17] S. Labeit, B. Kolmerer, and W. A. Linke, “The giant protein titin: emerging roles in physiology and pathophysiology," Circulation Research, vol. 80, no. 2, pp. 290-294, 1997.

[18] P. Fernando, J. S. Sandoz, W. Ding et al., "Bin 1 Src homology 3 domain acts as a scaffold for myofiber sarcomere assembly," The Journal of Biological Chemistry, vol. 284, no. 40, pp. 2767427686, 2009.

[19] R. J. Wechsler-Reya, K. J. Elliott, and G. C. Prendergast, "A role for the putative tumor suppressor Binl in muscle cell differentiation," Molecular and Cellular Biology, vol. 18, no. 1, pp. 566-575, 1998.

[20] N.-C. Mao, E. Steingrimsson, J. Duhadaway et al., "The murine Binl gene functions early in myogenesis and defines a new region of synteny between mouse chromosome 18 and human chromosome 2," Genomics, vol. 56, no. 1, pp. 51-58, 1999.

[21] A. J. Muller, J. F. Baker, J. B. DuHadaway et al., "Targeted disruption of the murine Bin1/Amphiphysin II gene does not disable endocytosis but results in embryonic cardiomyopathy with aberrant myofibril formation," Molecular and Cellular Biology, vol. 23, no. 12, pp. 4295-4306, 2003.

[22] S. Labeit, M. Gautel, A. Lakey, and J. Trinick, "Towards a molecular understanding of titin," The EMBO Journal, vol. 11, no. 5, pp. 1711-1716, 1992.

[23] S. Lange, F. Xiang, A. Yakovenko et al., "Cell biology: the kinase domain of titin controls muscle gene expression and protein turnover," Science, vol. 308, no. 5728, pp. 1599-1603, 2005.

[24] A. Kontrogianni-Konstantopoulos, D. H. Catino, J. C. Strong et al., "Obscurin modulates the assembly and organization of sarcomeres and the sarcoplasmic reticulum," The FASEB Journal, vol. 20, no. 12, pp. 2102-2111, 2006.

[25] A. Kontrogianni-Konstantopoulos, D. H. Catino, J. C. Strong, W. R. Randall, and R. J. Bloch, "Obscurin regulates the organization of myosin into A bands," American Journal of PhysiologyCell Physiology, vol. 287, no. 1, pp. C209-C217, 2004.

[26] A. B. Borisov, S. B. Sutter, A. Kontrogianni-Konstantopoulos, R. J. Bloch, M. V. Westfall, and M. W. Russell, "Essential role of obscurin in cardiac myofibrillogenesis and hypertrophic response: evidence from small interfering RNA-mediated gene silencing," Histochemistry and Cell Biology, vol. 125, no. 3, pp. 227-238, 2006.

[27] L.-Y. R. Hu and A. Kontrogianni-Konstantopoulos, "The kinase domains of obscurin interact with intercellular adhesion proteins," The FASEB Journal, vol. 27, no. 5, pp. 2001-2012, 2013.

[28] G. Xiong, H. Qadota, K. B. Mercer, L. A. McGaha, A. F. Oberhauser, and G. M. Benian, "A LIM-9 (FHL)/SCPL-1 (SCP) complex interacts with the $\mathrm{C}$-terminal protein kinase regions of UNC-89 (obscurin) in Caenorhabditis elegans muscle," Journal of Molecular Biology, vol. 386, no. 4, pp. 976-988, 2009.

[29] S. R. Cunha and P. J. Mohler, "Obscurin targets ankyrin-B and protein phosphatase 2A to the cardiac M-line," The Journal of Biological Chemistry, vol. 283, no. 46, pp. 31968-31980, 2008.

[30] N. Bhasin, S. R. Cunha, M. Mudannayake, M. S. Gigena, T. B. Rogers, and P. J. Mohler, "Molecular basis for PP2A regulatory subunit B56alpha targeting in cardiomyocytes," The American Journal of Physiology-Heart and Circulatory Physiology, vol. 293, no. 1, pp. H109-H119, 2007.
[31] H. Qadota, L. A. McGaha, K. B. Mercer, T. J. Stark, T. M. Ferrara, and G. M. Benian, "A novel protein phosphatase is a binding partner for the protein kinase domains of UNC-89 (obscurin) in Caenorhabditis elegans," Molecular Biology of the Cell, vol. 19, no. 6, pp. 2424-2432, 2008.

[32] W. M. J. Obermann, M. Gautel, K. Weber, and D. O. Fürst, "Molecular structure of the sarcomeric M band: mapping of titin and myosin binding domains in myomesin and the identification of a potential regulatory phosphorylation site in myomesin," The EMBO Journal, vol. 16, no. 2, pp. 211-220, 1997.

[33] A. Fukuzawa, S. Lange, M. Holt et al., "Interactions with titin and myomesin target obscurin and obscurin-like 1 to the Mband-Implications for hereditary myopathies," Journal of Cell Science, vol. 121, no. 11, pp. 1841-1851, 2008.

[34] D. Auerbach, S. Bantle, S. Keller et al., "Different domains of the $\mathrm{M}$-band protein myomesin are involved in myosin binding and M-band targeting," Molecular Biology of the Cell, vol. 10, no. 5, pp. 1297-1308, 1999.

[35] L. Tskhovrebova and J. Trinick, "Making muscle elastic: the structural basis of myomesin stretching," PLoS Biology, vol. 10, no. 2, Article ID e1001264, 2012.

[36] W. M. J. Obermann, U. Plessmann, K. Weber, and D. O. Furst, "Purification and biochemical characterization of myomesin, a myosin-binding and titin-binding protein, from bovine skeletal muscle," European Journal of Biochemistry, vol. 233, no. 1, pp. 110-115, 1995.

[37] J. Noguchi, M. Yanagisawa, M. Imamura et al., "Complete primary structure and tissue expression of chicken pectoralis M-protein," The Journal of Biological Chemistry, vol. 267, no. 28, pp. 20302-20310, 1992.

[38] F. Steiner, K. Weber, and D. O. Fürst, "Structure and expression of the gene encoding murine M-protein, a sarcomere-specific member of the immunoglobulin superfamily," Genomics, vol. 49, no. 1, pp. 83-95, 1998.

[39] B. K. Grove, L. Cerny, J. C. Perriard, and H. M. Eppenberger, "Myomesin and M-protein: expression of two M-band proteins in pectoral muscle and heart during development," Journal of Cell Biology, vol. 101, no. 4, pp. 1413-1421, 1985.

[40] B. K. Grove, B. Holmbom, and L. E. Thornell, "Myomesin and $M$ protein: differential expression in embryonic fibers during pectoral muscle development," Differentiation, vol. 34, no. 2, pp. 106-114, 1987.

[41] E. Carlsson, B. K. Grove, T. Wallimann, H. M. Eppenberger, and L.-E. Thornell, "Myofibrillar M-band proteins in rat skeletal muscles during development," Histochemistry, vol. 95, no. 1, pp. 27-35, 1990.

[42] W. M. J. Obermann, P. P. M. van der Ven, F. Steiner, K. Weber, and D. O. Fürst, "Mapping of a myosin-binding domain and a regulatory phosphorylation site in M-protein, a structural protein of the sarcomeric M band," Molecular Biology of the Cell, vol. 9, no. 4, pp. 829-840, 1998.

[43] H. C. A. Drexler, A. Ruhs, A. Konzer et al., "On marathons and Sprints: an integrated quantitative proteomics and transcriptomics analysis of differences between slow and fast muscle fibers," Molecular \& Cellular Proteomics, vol. 11, no. 6, Article ID M111.010801, 2012.

[44] M. A. Ackermann and A. Kontrogianni-Konstantopoulos, "Myosin binding protein-C slow: an intricate subfamily of proteins," Journal of Biomedicine and Biotechnology, vol. 2010, Article ID 652065, 10 pages, 2010.

[45] M. J. McGrath, D. L. Cottle, M.-A. Nguyen et al., "Four and a half LIM protein 1 binds myosin-binding protein $\mathrm{C}$ and 
regulates myosin filament formation and sarcomere assembly," The Journal of Biological Chemistry, vol. 281, no. 11, pp. 76667683, 2006.

[46] S. Lange, D. Auerbach, P. McLoughlin et al., "Subcellular targeting of metabolic enzymes to titin in heart muscle may be mediated by DRAL/FHL-2," Journal of Cell Science, vol. 115, no. 24, pp. 4925-4936, 2002.

[47] C. E. Torgan and M. P. Daniels, "Calcineurin localization in skeletal muscle offers insights into potential new targets," Journal of Histochemistry and Cytochemistry, vol. 54, no. 1, pp. 119-128, 2006.

[48] S. B. Geisler, D. Robinson, M. Hauringa et al., "Obscurin-like 1 , OBSL1, is a novel cytoskeletal protein related to obscurin," Genomics, vol. 89, no. 4, pp. 521-531, 2007.

[49] M. A. Ackermann and A. Kontrogianni-Konstantopoulos, "Myosin binding protein-C slow is a novel substrate for protein kinase A (PKA) and C (PKC) in skeletal muscle," Journal of Proteome Research, vol. 10, no. 10, pp. 4547-4555, 2011.

[50] P. E. Hoppe, J. Chau, K. A. Flanagan, A. R. Reedy, and L. A. Schriefer, "Caenorhabditis elegans unc-82 encodes a serine/threonine kinase important for myosin filament organization in muscle during growth," Genetics, vol. 184, no. 1, pp. 79-90, 2010.

[51] H. J. Koh, T. Toyoda, N. Fujii et al., "Sucrose nonfermenting AMPK-related kinase (SNARK) mediates contractionstimulated glucose transport in mouse skeletal muscle," Proceedings of the National Academy of Sciences of the United States of America, vol. 107, no. 35, pp. 15541-15546, 2010.

[52] F. Inazuka, N. Sugiyama, M. Tomita, T. Abe, G. Shioi, and H. Esumi, "Muscle-specific knock-out of NUAK family SNF1like kinase 1 (NUAK1) prevents high fat diet-induced glucose intolerance," The Journal of Biological Chemistry, vol. 287, no. 20, pp. 16379-16389, 2012.

[53] K. B. Reddy, J. E. B. Fox, M. G. Price et al., "Nuclear localization of myomesin-1: possible functions," Journal of Muscle Research and Cell Motility, vol. 29, no. 1, pp. 1-8, 2008.

[54] X. Wang, X. Liu, S. Wang, and K. Luan, "Myofibrillogenesis regulator 1 induces hypertrophy by promoting sarcomere organization in neonatal rat cardiomyocytes," Hypertension Research, vol. 35, no. 6, pp. 597-603, 2012.

[55] T. B. Li, X. H. Liu, S. Feng et al., "Characterization of MR-1, a novel myofibrillogenesis regulator in human muscle," Acta Biochimica et Biophysica Sinica (Shanghai), vol. 36, no. 6, pp. 412-418, 2004.

[56] H. L. Li, Z. G. She, T. B. Li et al., "Overexpression of myofibrillogenesis regulator-1 aggravates cardiac hypertrophy induced by angiotensin II in mice," Hypertension, vol. 49, no. 6, pp. 13991408, 2007.

[57] S. Just, B. Meder, I. M. Berger et al., "The myosin-interacting protein SMYD1 is essential for sarcomere organization," Journal of Cell Science, vol. 124, no. 18, pp. 3127-3136, 2011.

[58] J. Berkholz, L. Michalick, and B. Munz, "The E3 SUMO ligase Nse2 regulates sumoylation and nuclear-to-cytoplasmic translocation of skNAC-Smyd1 in myogenesis," Journal of Cell Science, vol. 127, part 17, pp. 3794-3804, 2014.

[59] I. Hwang and P. D. Gottlieb, "Bop: A new T-cell-restricted gene located upstream of and opposite to mouse CD8b," Immunogenetics, vol. 42, no. 5, pp. 353-361, 1995.

[60] I. Hwang and P. D. Gottlieb, "The Bop gene adjacent to the mouse CD8b gene encodes distinct zinc-finger proteins expressed in CTLs and in muscle," Journal of Immunology, vol. 158, no. 3, pp. 1165-1174, 1997.
[61] P. D. Gottlieb, S. A. Pierce, R. J. Sims III et al., "Bop encodes a muscle-restricted protein containing MYND and SET domains and is essential for cardiac differentiation and morphogenesis," Nature Genetics, vol. 31, no. 1, pp. 25-32, 2002.

[62] X. Tan, J. Rotllant, H. Li, P. DeDeyne, and S. J. Du, "SmyD1, a histone methyltransferase, is required for myofibril organization and muscle contraction in zebrafish embryos," Proceedings of the National Academy of Sciences of the United States of America, vol. 103 , no. 8, pp. 2713-2718, 2006.

[63] S. J. Du, X. Tan, and J. Zhang, "SMYD proteins: key regulators in skeletal and cardiac muscle development and function," The Anatomical Record, vol. 297, no. 9, pp. 1650-1662, 2014.

[64] R. J. Sims III, E. K. Weihe, L. Zhu, S. O’Malley, J. V. Harriss, and P. D. Gottlieb, "m-Bop, a repressor protein essential for cardiogenesis, interacts with skNAC, a heart- and muscle-specific transcription factor," The Journal of Biological Chemistry, vol. 277, no. 29, pp. 26524-26529, 2002.

[65] S. Lange, K. Ouyang, G. Meyer et al., "Obscurin determines the architecture of the longitudinal sarcoplasmic reticulum," Journal of Cell Science, vol. 122, no. 15, pp. 2640-2650, 2009.

[66] S. Lange, S. Perera, P. Teh, and J. Chen, "Obscurin and KCTD6 regulate cullin-dependent small ankyrin-1 (sAnk1.5) protein turnover," Molecular Biology of the Cell, vol. 23, no. 13, pp. 24902504, 2012.

[67] H. Iwabata, M. Yoshida, and Y. Komatsu, "Proteomic analysis of organ-specific post-translational lysine-acetylation and -methylation in mice by use of anti-acetyllysine and methyllysine mouse monoclonal antibodies," Proteomics, vol. 5, no. 18, pp. 4653-4664, 2005.

[68] S. Etienne-Manneville and A. Hall, "Rho GTPases in cell biology," Nature, vol. 420, no. 6916, pp. 629-635, 2002.

[69] I. de Curtis and J. Meldolesi, "Cell surface dynamics-how Rho GTPases orchestrate the interplay between the plasma membrane and the cortical cytoskeleton," Journal of Cell Science, vol. 125, no. 19, pp. 4435-4444, 2012.

[70] C. Bucci, P. Alifano, and L. Cogli, "The role of rab proteins in neuronal cells and in the trafficking of neurotrophin receptors," Membranes, vol. 4, no. 4, pp. 642-677, 2014.

[71] F. Nakamura, "FilGAP and its close relatives: a mediator of RhoRac antagonism that regulates cell morphology and migration," Biochemical Journal, vol. 453, no. 1, pp. 17-25, 2013.

[72] K. F. Wilson, J. W. Erickson, M. A. Antonyak, and R. A. Cerione, "Rho GTPases and their roles in cancer metabolism," Trends in Molecular Medicine, vol. 19, no. 2, pp. 74-82, 2013.

[73] R. García-Mata and K. Burridge, "Catching a GEF by its tail," Trends in Cell Biology, vol. 17, no. 1, pp. 36-43, 2007.

[74] G. Lazer and S. Katzav, "Guanine nucleotide exchange factors for RhoGTPases: good therapeutic targets for cancer therapy?" Cellular Signalling, vol. 23, no. 6, pp. 969-979, 2011.

[75] D. L. Ford-Speelman, J. A. Roche, A. L. Bowman, and R. J. Bloch, "The rho-guanine nucleotide exchange factor domain of obscurin activates rhoA signaling in skeletal muscle," Molecular Biology of the Cell, vol. 20, no. 17, pp. 3905-3917, 2009.

[76] H. Qadota, A. Blangy, G. Xiong, and G. M. Benian, “The $\mathrm{DH}-\mathrm{PH}$ region of the giant protein UNC-89 activates RHO-1 GTPase in Caenorhabditis elegans body wall muscle," Journal of Molecular Biology, vol. 383, no. 4, pp. 747-752, 2008.

[77] P. Madaule, T. Furuyashiki, T. Reid et al., "A novel partner for the GTP-bound forms of rho and rac," FEBS Letters, vol. 377, no. 2, pp. 243-248, 1995. 
[78] P. Madaule, M. Eda, N. Watanabe et al., "Role of citron kinase as a target of the small GTPase Rho in cytokinesis," Nature, vol. 394, no. 6692, pp. 491-494, 1998.

[79] X. Mu, A. Usas, Y. Tang et al., "RhoA mediates defective stem cell function and heterotopic ossification in dystrophic muscle of mice," The FASEB Journal, vol. 27, no. 9, pp. 3619-3631, 2013.

[80] M. Bowerman, L. M. Murray, J. G. Boyer, C. L. Anderson, and R. Kothary, "Fasudil improves survival and promotes skeletal muscle development in a mouse model of spinal muscular atrophy," BMC Medicine, vol. 10, article 24, 2012.

[81] H. Aoki, S. Izumo, and J. Sadoshima, "Angiotensin II activates RhoA in cardiac myocytes: a critical role of RhoA in angiotensin II-induced premyofibril formation," Circulation Research, vol. 82, no. 6, pp. 666-676, 1998.

[82] M. Hoshijima, V. P. Sah, Y. Wang, K. R. Chien, and J. H. Brown, "The low molecular weight GTPase Rho regulates myofibril formation and organization in neonatal rat ventricular myocytes: Involvement of Rho kinase," The Journal of Biological Chemistry, vol. 273, no. 13, pp. 7725-7730, 1998.

[83] J. H. Brown, D. P. Del Re, and M. A. Sussman, "The Rac and Rho hall of fame: a decade of hypertrophic signaling hits," Circulation Research, vol. 98, no. 6, pp. 730-742, 2006.

[84] S. Miyamoto, D. P. del Re, S. Y. Xiang, X. Zhao, G. Florholmen, and J. H. Brown, "Revisited and revised: is RhoA always a villain in cardiac pathophysiology?" Journal of Cardiovascular Translational Research, vol. 3, no. 4, pp. 330-343, 2010.

[85] S. Y. Xiang, D. Vanhoutte, D. P. del Re et al., "RhoA protects the mouse heart against ischemia/reperfusion injury," The Journal of Clinical Investigation, vol. 121, no. 8, pp. 3269-3276, 2011.

[86] D. P. del Re, S. Miyamoto, and J. H. Brown, "RhoA/Rho kinase up-regulate Bax to activate a mitochondrial death pathway and induce cardiomyocyte apoptosis," The Journal of Biological Chemistry, vol. 282, no. 11, pp. 8069-8078, 2007.

[87] D. P. del Re, S. Miyamoto, and J. H. Brown, "Focal adhesion kinase as a RhoA-activable signaling scaffold mediating akt activation and cardiomyocyte protection," The Journal of Biological Chemistry, vol. 283, no. 51, pp. 35622-35629, 2008.

[88] A. Zeidan, X. T. Gan, A. Thomas, and M. Karmazyn, "Prevention of RhoA activation and cofilin-mediated actin polymerization mediates the antihypertrophic effect of adenosine receptor agonists in angiotensin II- and endothelin-1-treated cardiomyocytes," Molecular and Cellular Biochemistry, vol. 385, no. 1-2, pp. 239-248, 2014.

[89] D. Frank, C. Kuhn, B. Brors et al., "Gene expression pattern in biomechanically stretched cardiomyocytes: evidence for a stretch-specific gene program," Hypertension, vol. 51, no. 2, pp. 309-318, 2008.

[90] A. M. de Jong, A. H. Maass, S. U. Oberdorf-Maass, R. A. de Boer, W. H. van Gilst, and I. C. van Gelder, "Cyclical stretch induces structural changes in atrial myocytes," Journal of Cellular and Molecular Medicine, vol. 17, no. 6, pp. 743-753, 2013.

[91] M. Hoshijima, "Mechanical stress-strain sensors embedded in cardiac cytoskeleton: Z disk, titin, and associated structures," The American Journal of Physiology-Heart and Circulatory Physiology, vol. 290, no. 4, pp. H1313-H1325, 2006.

[92] R. Knöll and S. Marston, "On mechanosensation, acto/myosin interaction, and hypertrophy," Trends in Cardiovascular Medicine, vol. 22, no. 1, pp. 17-22, 2012.

[93] E. M. Puchner, A. Alexandrovich, L. K. Ay et al., "Mechanoenzymatics of titin kinase," Proceedings of the National Academy of Sciences of the United States of America, vol. 105, no. 36, pp. 13385-13390, 2008.
[94] V. Kirkin, T. Lamark, Y. S. Sou et al., "A role for NBR1 in autophagosomal degradation of ubiquitinated substrates," Molecular Cell, vol. 33, no. 4, pp. 505-516, 2009.

[95] V. I. Korolchuk, A. Mansilla, F. M. Menzies, and D. C. Rubinsztein, "Autophagy inhibition compromises degradation of ubiquitin-proteasome pathway substrates," Molecular Cell, vol. 33 , no. 4, pp. 517-527, 2009.

[96] N. Myeku and M. E. Figueiredo-Pereira, "Dynamics of the degradation of ubiquitinated proteins by proteasomes and autophagy: association with sequestosome 1/p62," The Journal of Biological Chemistry, vol. 286, no. 25, pp. 22426-22440, 2011.

[97] J. Bogomolovas, A. Gasch, F. Simkovic, D. J. Rigden, S. Labeit, and O. Mayans, "Titin kinase is an inactive pseudokinase scaffold that supports MuRF1 recruitment to the sarcomeric Mline," Open Biology, vol. 4, no. 5, Article ID 140041, 2014.

[98] C. Batters, C. Veigel, E. Homsher, and J. R. Sellers, “To understand muscle you must take it apart," Frontiers in Physiology, vol. 5, no. 90, 2014.

[99] T. Clausen, "Quantification of $\mathrm{Na}^{+}, \mathrm{K}^{+}$pumps and their transport rate in skeletal muscle: functional significance," Journal of General Physiology, vol. 142, no. 4, pp. 327-345, 2013.

[100] A. R. Marks, "Calcium cycling proteins and heart failure: mechanisms and therapeutics," The Journal of Clinical Investigation, vol. 123, no. 1, pp. 46-52, 2013.

[101] C. R. Hancock, J. J. Brault, and R. L. Terjung, "Protecting the cellular energy state during contractions: role of AMP deaminase," Journal of Physiology and Pharmacology, vol. 57, no. 10, pp. 17-29, 2006.

[102] T. Wallimann, M. Tokarska-Schlattner, and U. Schlattner, "The creatine kinase system and pleiotropic effects of creatine." Amino Acids, vol. 40, no. 5, pp. 1271-1296, 2011.

[103] R. M. Payne, R. C. Haas, and A. W. Strauss, "Structural characterization and tissue-specific expression of the mRNAs encoding isoenzymes from two rat mitochondrial creatine kinase genes," Biochim Biophys Acta, vol. 1089, no. 3, pp. 352$361,1991$.

[104] T. Wallimann and H. M. Eppenberger, "Localization and function of M-line-bound creatine kinase. M-band model and creatine phosphate shuttle," Cell and Muscle Motility, vol. 6, pp. 239-285, 1985.

[105] R. Ventura-Clapier, A. Kaasik, and V. Veksler, "Structural and functional adaptations of striated muscles to CK deficiency," Molecular and Cellular Biochemistry, vol. 256-257, no. 1-2, pp. 29-41, 2004.

[106] T. Hornemann, S. Kempa, M. Himmel, K. Hayeß, D. O. Fürst, and T. Wallimann, "Muscle-type creatine kinase interacts with central domains of the M-band proteins myomesin and Mprotein," Journal of Molecular Biology, vol. 332, no. 4, pp. 877887, 2003.

[107] J. van Deursen, A. Heerschap, F. Oerlemans et al., "Skeletal muscles of mice deficient in muscle creatine kinase lack burst activity," Cell, vol. 74, no. 4, pp. 621-631, 1993.

[108] J. van Deursen, W. Ruitenbeek, A. Heerschap, P. Jap, H. Ter Laak, and B. Wieringa, "Creatine kinase (CK) in skeletal muscle energy metabolism: a study of mouse mutants with graded reduction in muscle CK expression," Proceedings of the National Academy of Sciences of the United States of America, vol. 91, no. 19, pp. 9091-9095, 1994.

[109] I. Momken, P. Lechêne, N. Koulmann et al., "Impaired voluntary running capacity of creatine kinase-deficient mice," Journal of Physiology, vol. 565, no. 3, pp. 951-964, 2005. 
[110] S. Patra, S. Bera, S. SinhaRoy et al., "Progressive decrease of phosphocreatine, creatine and creatine kinase in skeletal muscle upon transformation to sarcoma," FEBS Journal, vol. 275, no. 12, pp. 3236-3247, 2008.

[111] D. G. Hardie, "Metabolic control: a new solution to an old problem," Current Biology, vol. 10, no. 20, pp. R757-R759, 2000.

[112] C. R. Hancock, E. Janssen, and R. L. Terjung, "Skeletal muscle contractile performance and ADP accumulation in adenylate kinase-deficient mice," The American Journal of PhysiologyCell Physiology, vol. 288, no. 6, pp. C1287-C1297, 2005.

[113] C. Panayiotou, N. Solaroli, and A. Karlsson, "The many isoforms of human adenylate kinases," International Journal of Biochemistry and Cell Biology, vol. 49, no. 1, pp. 75-83, 2014.

[114] E. Janssen, P. P. Dzeja, F. Oerlemans et al., "Adenylate kinase 1 gene deletion disrupts muscle energetic economy despite metabolic rearrangement," The EMBO Journal, vol. 19, no. 23, pp. 6371-6381, 2000.

[115] D. Pucar, E. Janssen, P. P. Dzeja et al., "Compromised energetics in the adenylate kinase AK1 gene knockout heart under metabolic stress," The Journal of Biological Chemistry, vol. 275, no. 52, pp. 41424-41429, 2000.

[116] D. Pucar, P. Bast, R. J. Gumina et al., "Adenylate kinase AK1 knockout heart: energetics and functional performance under ischemia-reperfusion," The American Journal of PhysiologyHeart and Circulatory Physiology, vol. 283, no. 2, pp. H776H782, 2002.

[117] R. L. Sabina and D. K. Mahnke-Zizelman, “Towards an understanding of the functional significance of $\mathrm{N}$-terminal domain divergence in human AMP deaminase isoforms," Pharmacology and Therapeutics, vol. 87, no. 2-3, pp. 279-283, 2000.

[118] N. Ogasawara, H. Goto, Y. Yamada, and T. Watanabe, "Distribution of AMP-deaminase isozymes in rat tissues," European Journal of Biochemistry, vol. 87, no. 2, pp. 297-304, 1978.

[119] N. Ogasawara, H. Goto, Y. Yamada, T. Watanabe, and T. Asano, "AMP deaminase isozymes in human tissues," Biochim Biophys Acta: General Subjects, vol. 714, no. 2, pp. 298-306, 1982.

[120] T. H. van Kuppevelt, J. H. Veerkamp, W. N. Fishbein, N. Ogasawara, and R. L. Sabina, "Immunolocalization of AMPdeaminase isozymes in human skeletal muscle and cultured muscle cells: Concentration of isoform $\mathrm{M}$ at the neuromuscular junction," Journal of Histochemistry and Cytochemistry, vol. 42, no. 7, pp. 861-868, 1994.

[121] K. Uyeda, "Phosphofructokinase," Advances in Enzymology and Related Areas of Molecular Biology, vol. 48, pp. 193-244, 1979.

[122] G. A. Dunaway, "A review of animal phosphofructokinase isozymes with an emphasis on their physiological role," Molecular and Cellular Biochemistry, vol. 52, no. 1, pp. 75-91, 1983.

[123] G. A. Dunaway, T. P. Kasten, T. Sebo, and R. Trapp, "Analysis of the phosphofructokinase subunits and isoenzymes in human tissues," Biochemical Journal, vol. 251, no. 3, pp. 677-683, 1988.

[124] M. Sola-Penna, D. Da Silva, W. S. Coelho, M. M. MarinhoCarvalho, and P. Zancan, "Regulation of mammalian muscle type 6-phosphofructo-1-kinase and its implication for the control of the metabolism," IUBMB Life, vol. 62, no. 11, pp. 791-796, 2010.

[125] E. Van Schaftingen, L. Hue, and H. G. Hers, "Fructose 2,6bisphosphate, the probable structure of the glucose- and glucagon-sensitive stimulator of phosphofructokinase," Biochemical Journal, vol. 192, no. 3, pp. 897-901, 1980.
[126] T. Schöneberg, M. Kloos, A. Brüser, J. Kirchberger, and N. Sträter, "Structure and allosteric regulation of eukaryotic 6phosphofructokinases," Biological Chemistry, vol. 394, no. 8, pp. 977-993, 2013.

[127] P. B. Garland, P. J. Randle, and E. A. Newsholme, "Citrate as an intermediary in the inhibition of phosphofructokinase in rat heart muscle by fatty acids, ketone bodies, pyruvate, diabetes and starvation," Nature, vol. 200, no. 4902, pp. 169-170, 1963.

[128] M. Y. Tsai and R. G. Kemp, "Rabbit brain phosphofructokinase. Comparison of regulatory properties with those of other phosphofructokinase isozymes," Journal of Biological Chemistry, vol. 249, no. 20, pp. 6590-6596, 1974.

[129] A. M. Mahrenholz, L. Lan, and T. E. Mansour, "Phosphorylation of heart phosphofructokinase by $\mathrm{Ca}^{2+} /$ calmodulin protein kinase," Biochemical and Biophysical Research Communications, vol. 174, no. 3, pp. 1255-1259, 1991.

[130] C. M. Jenkins, J. Yang, H. F. Sims, and R. W. Gross, "Reversible high affinity inhibition of phosphofructokinase-1 by Acyl-CoA: a mechanism integrating glycolytic flux with lipid metabolism," Journal of Biological Chemistry, vol. 286, no. 14, pp. 11937-11950, 2011.

[131] P. Zancan, F. V. R. Almeida, J. Faber-Barata, J. M. Dellias, and M. Sola-Penna, "Fructose-2,6-bisphosphate counteracts guanidinium chloride-, thermal-, and ATP-induced dissociation of skeletal muscle key glycolytic enzyme 6-phosphofructo1-kinase: a structural mechanism for PFK allosteric regulation," Archives of Biochemistry and Biophysics, vol. 467, no. 2, pp. 275282, 2007.

[132] P. Zancan, M. M. Marinho-Carvalho, J. Faber-Barata, J. M. M. Dellias, and M. Sola-Penna, "ATP and fructose2,6-bisphosphate regulate skeletal muscle 6-phosphofructo-1kinase by altering its quaternary structure," IUBMB Life, vol. 60, no. 8, pp. 526-533, 2008.

[133] R.-S. Liou and S. Anderson, "Activation of rabbit muscle phosphofructokinase by F-actin and reconstituted thin filaments," Biochemistry, vol. 19, no. 12, pp. 2684-2688, 1980.

[134] S. J. Roberts and G. N. Somero, "Binding of phosphofructokinase to filamentous actin," Biochemistry, vol. 26, no. 12, pp. 3437-3442, 1987.

[135] C.-Z. Malca, T. Livnat, and R. Beitner, "Insulin rapidly stimulates binding of phosphofructokinase and aldolase to muscle cytoskeleton," International Journal of Biochemistry, vol. 24, no. 5, pp. 821-826, 1992.

[136] J. P. J. Schmitz, W. Groenendaal, B. Wessels et al., "Combined in vivo and in silico investigations of activation of glycolysis in contracting skeletal muscle," The American Journal of Physiology-Cell Physiology, vol. 304, no. 2, pp. C180-C193, 2013.

[137] B. Buschmeier, H. E. Meyer, and G. W. Mayr, "Characterization of the calmodulin-binding sites of muscle phosphofructokinase and comparison with known calmodulin-binding domains," Journal of Biological Chemistry, vol. 262, no. 20, pp. 9454-9462, 1987.

[138] M. M. Marinho-Carvalho, P. V. Costa-Mattos, G. A. Spitz, P. Zancan, and M. Sola-Penna, "Calmodulin upregulates skeletal muscle 6-phosphofructo-1-kinase reversing the inhibitory effects of allosteric modulators," Biochimica et Biophysica Acta, vol. 1794, no. 8, pp. 1175-1180, 2009.

[139] M. García, A. Pujol, A. Ruzo et al., "Phosphofructo-1-kinase deficiency leads to a severe cardiac and hematological disorder in addition to skeletal muscle glycogenosis," PLoS Genetics, vol. 5, no. 8, Article ID e1000615, 2009. 
[140] G. Foucault, M. Vacher, T. Merkulova, A. Keller, and M. ArrioDupont, "Presence of enolase in the M-band of skeletal muscle and possible indirect interaction with the cytosolic muscle isoform of creatine kinase," Biochemical Journal, vol. 338, no. 1, pp. 115-121, 1999.

[141] A. Keller, J. Demeurie, T. Merkulova et al., "Fibre-type distribution and subcellular localisation of $\alpha$ and $\beta$ enolase in mouse striated muscle," Biology of the Cell, vol. 92, no. 7, pp. 527-535, 2000.

[142] S. P. Craig, I. N. M. Day, R. J. Thompson, and I. W. Craig, "Localisation of neurone-specific enolase (ENO2) to 12p13," Cytogenetics and Cell Genetics, vol. 54, no. 1-2, pp. 71-73, 1990.

[143] S. Feo, D. Oliva, G. Barbieri, W. Xu, M. Fried, and A. Giallongo, "The gene for the muscle-specific enolase is on the short arm of human chromosome 17," Genomics, vol. 6, no. 1, pp. 192-194, 1990.

[144] C. C. Rider and C. B. Taylor, "Enolase isoenzymes in rat tissues. Electrophoretic, chromatographic, immunological and kinetic properties," Biochimica et Biophysica Acta, vol. 365, no. 1, pp. 285-300, 1974.

[145] C. C. Rider and C. B. Taylor, "Enolase isoenzymes. II. Hybridization studies, developmental and phylogenetic aspects," Biochimica et Biophysica Acta, vol. 405, no. 1, pp. 175-187, 1975.

[146] O. F. Wolf, "Enolase," in The Enzymes, pp. 499-538, Academic Press, New York, NY, USA, 1971.

[147] T. Ibi, K. Sahashi, K. Kato, A. Takahashi, and I. Sobue, "Immunohistochemical demonstration of $\beta$-enolase in human skeletal muscle," Muscle \& Nerve, vol. 6, no. 9, pp. 661-663, 1983.

[148] K. Kato, A. Shimizu, R. Semba, and T. Satoh, "Tissue distribution, developmental profiles and effect of denervation of enolase isozymes in rat muscles," Biochimica et Biophysica Acta, vol. 841, no. 1, pp. 50-58, 1985.

[149] A. Keller, J.-D. Rouzeau, F. Farhadian et al., "Differential expression of $\alpha$ - and $\beta$-enolase genes during rat heart development and hypertrophy," The American Journal of Physiology-Heart and Circulatory Physiology, vol. 269, no. 6, pp. H1843-H1851, 1995.

[150] H. S. Leong, M. Grist, H. Parsons et al., "Accelerated rates of glycolysis in the hypertrophied heart: are they a methodological artifact?" American Journal of Physiology-Endocrinology and Metabolism, vol. 282, no. 5, pp. E1039-E1045, 2002.

[151] L.-A. Zhu, N.-Y. Fang, P.-J. Gao, X. Jin, and H.-Y. Wang, "Differential expression of $\alpha$-enolase in the normal and pathological cardiac growth," Experimental and Molecular Pathology, vol. 87, no. 1, pp. 27-31, 2009.

[152] X. Jin, L.-S. Wang, L. Xia et al., "Hyper-phosphorylation of $\alpha$-enolase in hypertrophied left ventricle of spontaneously hypertensive rat," Biochemical and Biophysical Research Communications, vol. 371, no. 4, pp. 804-809, 2008.

[153] A. Shimizu, F. Suzuki, and K. Kato, "Characterization of $\alpha \alpha, \beta \beta$, $\gamma \gamma$ and $\alpha \gamma$ human enolase isozymes, and preparation of hybrid enolases $(\alpha \gamma, \beta \gamma$ and $\alpha \beta)$ from homodimeric forms," Biochimica et Biophysica Acta, vol. 748, no. 2, pp. 278-284, 1983.

[154] Y. Mizukami, A. Iwamatsu, T. Aki et al., "ERK1/2 regulates intracellular ATP levels through $\alpha$-enolase expression in cardiomyocytes exposed to ischemic hypoxia and reoxygenation," The Journal of Biological Chemistry, vol. 279, no. 48, pp. 5012050131, 2004.

[155] T. Merkulova, M. Dehaupas, M.-C. Nevers, C. Créminon, H. Alameddine, and A. Keller, "Differential modulation of $\alpha, \beta$ and $\gamma$ enolase isoforms in regenerating mouse skeletal muscle,"
European Journal of Biochemistry, vol. 267, no. 12, pp. 3735-3743, 2000.

[156] À. Díaz-Ramos, A. Roig-Borrellas, A. García-Melero, and R. López-Alemany, " $\alpha$-enolase, a multifunctional protein: Its role on pathophysiological situations," Journal of Biomedicine and Biotechnology, vol. 2012, Article ID 156795, 12 pages, 2012.

[157] S. H. Witt, H. Granzier, C. C. Witt, and S. Labeit, "MURF-1 and MURF-2 target a specific subset of myofibrillar proteins redundantly: towards understanding MURF-dependent muscle ubiquitination," Journal of Molecular Biology, vol. 350, no. 4, pp. 713-722, 2005.

[158] J. Fielitz, M.-S. Kim, J. M. Shelton et al., "Myosin accumulation and striated muscle myopathy result from the loss of muscle RING finger 1 and 3," The Journal of Clinical Investigation, vol. 117, no. 9, pp. 2486-2495, 2007.

[159] S. Koyama, S. Hata, C. C. Witt et al., "Muscle RING-finger protein-1 (MuRF1) as a connector of muscle energy metabolism and protein synthesis," Journal of Molecular Biology, vol. 376, no. 5, pp. 1224-1236, 2008.

[160] T.-J. Zhao, Y.-B. Yan, Y. Liu, and H.-M. Zhou, “The generation of the oxidized form of creatine kinase is a negative regulation on muscle creatine kinase," The Journal of Biological Chemistry, vol. 282, no. 16, pp. 12022-12029, 2007.

[161] J. A. Spencer, S. Eliazer, R. L. Ilaria Jr., J. A. Richardson, and E. N. Olson, "Regulation of microtubule dynamics and myogenic differentiation by MURF, a striated muscle RINGfinger protein," Journal of Cell Biology, vol. 150, no. 4, pp. 771784, 2000.

[162] S. C. Bodine, E. Latres, S. Baumhueter et al., "Identification of ubiquitin ligases required for skeletal muscle atrophy," Science, vol. 294, no. 5547, pp. 1704-1708, 2001.

[163] T. Centner, J. Yano, E. Kimura et al., "Identification of muscle specific ring finger proteins as potential regulators of the titin kinase domain," Journal of Molecular Biology, vol. 306, no. 4, pp. 717-726, 2001.

[164] J. Callis, "The ubiquitination machinery of the ubiquitin system," The Arabidopsis Book, vol. 12, article e0174, 2014.

[165] A. S. Moriscot, I. L. Baptista, J. Bogomolovas et al., "MuRF1 is a muscle fiber-type II associated factor and together with MuRF2 regulates type-II fiber trophicity and maintenance," Journal of Structural Biology, vol. 170, no. 2, pp. 344-353, 2010.

[166] S. Perera, B. Mankoo, and M. Gautel, "Developmental regulation of MURF E3 ubiquitin ligases in skeletal muscle," Journal of Muscle Research and Cell Motility, vol. 33, no. 2, pp. 107-122, 2012.

[167] C. C. Gregorio, C. N. Perry, and A. S. Mcelhinny, "Functional properties of the titin/connectin-associated proteins, the muscle-specific RING finger proteins (MURFs), in striated muscle," Journal of Muscle Research and Cell Motility, vol. 26, no. 6-8, pp. 389-400, 2005.

[168] A. S. McElhinny, K. Kakinuma, H. Sorimachi, S. Labeit, and C. C. Gregorio, "Muscle-specific RING finger-1 interacts with titin to regulate sarcomeric M-line and thick filament structure and may have nuclear functions via its interaction with glucocorticoid modulatory element binding protein-1," The Journal of Cell Biology, vol. 157, no. 1, pp. 125-136, 2002.

[169] A. S. McElhinny, C. N. Perry, C. C. Witt, S. Labeit, and C. C. Gregorio, "Muscle-specific RING finger-2 (MURF-2) is important for microtubule, intermediate filament and sarcomeric M-line maintenance in striated muscle development," Journal of Cell Science, vol. 117, part 15, pp. 3175-3188, 2004. 
[170] M. S. Willis, C. Ike, L. Li, D.-Z. Wang, D. J. Glass, and C. Patterson, "Muscle ring finger 1, but not muscle ring finger 2, regulates cardiac hypertrophy in vivo," Circulation Research, vol. 100, no. 4, pp. 456-459, 2007.

[171] C. C. Witt, S. H. Witt, S. Lerche, D. Labeit, W. Back, and S. Labeit, "Cooperative control of striated muscle mass and metabolism by MuRF1 and MuRF2," The EMBO Journal, vol. 27, no. 2, pp. 350-360, 2008.

[172] J. Fielitz, E. van Rooij, J. A. Spencer et al., "Loss of musclespecific RING-finger 3 predisposes the heart to cardiac rupture after myocardial infarction," Proceedings of the National Academy of Sciences of the United States of America, vol. 104, no. 11, pp. 4377-4382, 2007.

[173] A. L. Kho, S. Perera, A. Alexandrovich, and M. Gautel, "The sarcomeric cytoskeleton as a target for pharmacological intervention," Current Opinion in Pharmacology, vol. 12, no. 3, pp. 347-354, 2012.

[174] E. M. McNally, J. R. Golbus, and M. J. Puckelwartz, "Genetic mutations and mechanisms in dilated cardiomyopathy," The Journal of Clinical Investigation, vol. 123, no. 1, pp. 19-26, 2013.

[175] C. Chauveau, C. G. Bonnemann, C. Julien et al., "Recessive TTN truncating mutations define novel forms of core myopathy with heart disease," Human Molecular Genetics, vol. 23, no. 4, pp. 980-991, 2014.

[176] B. S. Cowling, D. L. Cottle, B. R. Wilding, C. E. D’Arcy, C. A. Mitchell, and M. J. McGrath, "Four and a half LIM protein 1 gene mutations cause four distinct human myopathies: a comprehensive review of the clinical, histological and pathological features," Neuromuscular Disorders, vol. 21, no. 4, pp. 237-251, 2011.

[177] F. W. Friedrich, B. R. Wilding, S. Reischmann et al., "Evidence for FHL1 as a novel disease gene for isolated hypertrophic cardiomyopathy," Human Molecular Genetics, vol. 21, no. 14, pp. 3237-3254, 2012.

[178] D. Selcen, M. B. Bromberg, S. S. Chin, and A. G. Engel, "Reducing bodies and myofibrillar myopathy features in FHL1 muscular dystrophy," Neurology, vol. 77, no. 22, pp. 1951-1959, 2011.

[179] S. Shalaby, Y. K. Hayashi, I. Nonaka, S. Noguchi, and I. Nishino, "Novel FHL1 mutations in fatal and benign reducing body myopathy," Neurology, vol. 72, no. 4, pp. 375-376, 2009.

[180] L. B. Waddell, J. Tran, X. F. Zheng et al., "A study of FHL1, BAG3, MATR3, PTRF and TCAP in Australian muscular dystrophy patients," Neuromuscular Disorders, vol. 21, no. 11, pp. 776-781, 2011.

[181] L. Gueneau, A. T. Bertrand, J.-P. Jais et al., "Mutations of the FHL1 gene cause Emery-Dreifuss muscular dystrophy," American Journal of Human Genetics, vol. 85, no. 3, pp. 338-353, 2009.

[182] D.-H. Chen, W. H. Raskind, W. W. Parson et al., "A novel mutation in FHL1 in a family with X-linked scapuloperoneal myopathy: phenotypic spectrum and structural study of FHL1 mutations," Journal of the Neurological Sciences, vol. 296, no. 1-2, pp. 22-29, 2010.

[183] C. M. Quinzii, T. H. Vu, K. C. Min et al., "X-linked dominant scapuloperoneal myopathy is due to a mutation in the gene encoding four-and-a-half-LIM protein 1," The American Journal of Human Genetics, vol. 82, no. 1, pp. 208-213, 2008.

[184] J. Schessl, A. L. Taratuto, C. Sewry et al., "Clinical, histological and genetic characterization of reducing body myopathy caused by mutations in FHL1," Brain, vol. 132, no. 2, pp. 452-464, 2009.
[185] J. Schessl, A. Columbus, Y. Hu et al., "Familial reducing body myopathy with cytoplasmic bodies and rigid spine revisited: identification of a second LIM domain mutation in FHL1," Neuropediatrics, vol. 41, no. 1, pp. 43-46, 2010.

[186] J. Schessl, Y. Zou, M. J. McGrath et al., "Proteomic identification of FHL1 as the protein mutated in human reducing body myopathy," Journal of Clinical Investigation, vol. 118, no. 3, pp. 904-912, 2008.

[187] C. Windpassinger, B. Schoser, V. Straub et al., "An X-linked myopathy with postural muscle atrophy and generalized hypertrophy, termed XMPMA, is caused by mutations in FHL1," The American Journal of Human Genetics, vol. 82, no. 1, pp. 88-99, 2008.

[188] T. Schreckenbach, W. Henn, W. Kress et al., "Novel FHL1 mutation in a family with reducing body myopathy," Muscle and Nerve, vol. 47, no. 1, pp. 127-134, 2013.

[189] H. R. Tiffin, Z. A. Jenkins, M. J. Gray et al., "Dysregulation of FHL1 spliceforms due to an indel mutation produces an EmeryDreifuss muscular dystrophy plus phenotype," Neurogenetics, vol. 14, no. 2, pp. 113-121, 2013.

[190] B. Schoser, H. H. Goebel, I. Janisch et al., "Consequences of mutations within the C terminus of the FHL1 gene," Neurology, vol. 73, no. 7, pp. 543-551, 2009.

[191] T. D. Gossios, L. R. Lopes, and P. M. Elliott, "Left ventricular hypertrophy caused by a novel nonsense mutation in FHL1," European Journal of Medical Genetics, vol. 56, no. 5, pp. 251-255, 2013.

[192] H. Hartmannova, M. Kubanek, M. Sramko et al., "Isolated Xlinked hypertrophic cardiomyopathy caused by a novel mutation of the four-and-a-half LIM domain 1 gene," Circulation: Cardiovascular Genetics, vol. 6, no. 6, pp. 543-551, 2013.

[193] H. Knoblauch, C. Geier, S. Adams et al., "Contractures and hypertrophic cardiomyopathy in a novel FHL1 mutation," Annals of Neurology, vol. 67, no. 1, pp. 136-140, 2010.

[194] T. Arimura, T. Hayashi, Y. Matsumoto et al., "Structural analysis of four and half LIM protein-2 in dilated cardiomyopathy," Biochemical and Biophysical Research Communications, vol. 357, no. 1, pp. 162-167, 2007.

[195] C. A. Gurnett, D. M. Desruisseau, K. McCall et al., "Myosin binding protein $\mathrm{Cl}$ : a novel gene for autosomal dominant distal arthrogryposis type 1," Human Molecular Genetics, vol. 19, no. 7, Article ID ddp587, pp. 1165-1173, 2010.

[196] B. Markus, G. Narkis, D. Landau, R. Z. Birk, I. Cohen, and O. S. Birk, "Autosomal recessive lethal congenital contractural syndrome type 4 (LCCS4) caused by a mutation in MYBPC1," Human Mutation, vol. 33, no. 10, pp. 1435-1438, 2012.

[197] R. M. Toydemir, A. Rutherford, F. G. Whitby, L. B. Jorde, J. C. Carey, and M. J. Bamshad, "Mutations in embryonic myosin heavy chain (MYH3) cause Freeman-Sheldon syndrome and Sheldon-Hall syndrome," Nature Genetics, vol. 38, no. 5, pp. 561$565,2006$.

[198] E. Carniel, M. R. G. Taylor, G. Sinagra et al., “ $\alpha$-myosin heavy chain: a sarcomeric gene associated with dilated and hypertrophic phenotypes of cardiomyopathy," Circulation, vol. 112, no. 1, pp. 54-59, 2005.

[199] J. T. Granados-Riveron, T. K. Ghosh, M. Pope et al., “ $\alpha$-Cardiac myosin heavy chain (MYH6) mutations affecting myofibril formation are associated with congenital heart defects," Human Molecular Genetics, vol. 19, no. 20, pp. 4007-4016, 2010.

[200] S. L. van Driest, M. A. Jaeger, S. R. Ommen et al., "Comprehensive analysis of the beta-myosin heavy chain gene in 389 
unrelated patients with hypertrophic cardiomyopathy," Journal of the American College of Cardiology, vol. 44, no. 3, pp. 602-610, 2004.

[201] P. Richard, P. Charron, L. Carrier et al., "Hypertrophic cardiomyopathy: distribution of disease genes, spectrum of mutations, and implications for a molecular diagnosis strategy," Circulation, vol. 107, no. 17, pp. 2227-2232, 2003.

[202] R. R. Tanjore, A. D. Sikindlapuram, N. Calambur, B. Thakkar, P. G. Kerkar, and P. Nallari, "Genotype-phenotype correlation of R870H mutation in hypertrophic cardiomyopathy," Clinical Genetics, vol. 69, no. 5, pp. 434-436, 2006.

[203] M. Arad, M. Penas-Lado, L. Monserrat et al., "Gene mutations in apical hypertrophic cardiomyopathy," Circulation, vol. 112, no. 18, pp. 2805-2811, 2005.

[204] L. Fananapazir, M. C. Dalakas, F. Cyran, G. Cohn, and N. D. Epstein, "Missense mutations in the $\beta$-myosin heavy-chain gene cause central core disease in hypertrophic cardiomyopathy," Proceedings of the National Academy of Sciences of the United States of America, vol. 90, no. 9, pp. 3993-3997, 1993.

[205] H. Watkins, L. Thierfelder, D.-S. Hwang, W. McKenna, J. G. Seidman, and C. E. Seidman, "Sporadic hypertrophic cardiomyopathy due to de novo myosin mutations," Journal of Clinical Investigation, vol. 90, no. 5, pp. 1666-1671, 1992.

[206] M. Michels, O. I. I. Soliman, J. Phefferkorn et al., "Disease penetrance and risk stratification for sudden cardiac death in asymptomatic hypertrophic cardiomyopathy mutation carriers," European Heart Journal, vol. 30, no. 21, pp. 2593-2598, 2009.

[207] H. Nishi, A. Kimura, H. Harada et al., "Possible gene dose effect of a mutant cardiac $\beta$-myosin heavy chain gene on the clinical expression of familial hypertrophic cardiomyopathy," Biochemical and Biophysical Research Communications, vol. 200, no. 1, pp. 549-556, 1994.

[208] E. Villard, L. Duboscq-Bidot, P. Charron et al., "Mutation screening in dilated cardiomyopathy: prominent role of the beta myosin heavy chain gene," European Heart Journal, vol. 26, no. 8, pp. 794-803, 2005.

[209] P. Jääskeläinen, T. Heliö, K. Aalto-Setälä et al., "A new common mutation in the cardiac beta-myosin heavy chain gene in Finnish patients with hypertrophic cardiomyopathy," Annals of Medicine, vol. 46, no. 6, pp. 424-429, 2014.

[210] L. Hougs, O. Havndrup, H. Bundgaard et al., "One third of Danish hypertrophic cardiomyopathy patients with MYH7 mutations have mutations [corrected] in MYH7 rod region," European Journal of Human Genetics, vol. 13, no. 2, pp. 161-165, 2005.

[211] A. Perrot, H. Schmidt-Traub, B. Hoffmann et al., "Prevalence of cardiac beta-myosin heavy chain gene mutations in patients with hypertrophic cardiomyopathy," Journal of Molecular Medicine, vol. 83, no. 6, pp. 468-477, 2005.

[212] J.-M. Park, Y. J. Kim, J. H. Yoo et al., "A novel MYH7 mutation with prominent paraspinal and proximal muscle involvement," Neuromuscular Disorders, vol. 23, no. 7, pp. 580-586, 2013.

[213] T. Cullup, P. J. Lamont, S. Cirak et al., "Mutations in MYH7 cause Multi-minicore Disease $(\mathrm{MmD})$ with variable cardiac involvement," Neuromuscular Disorders, vol. 22, no. 12, pp. 1096-1104, 2012.

[214] P. J. Lamont, W. Wallefeld, D. Hilton-Jones et al., "Novel mutations widen the phenotypic spectrum of slow skeletal/ $\beta$ cardiac myosin (MYH7) distal myopathy," Human Mutation, vol. 35, no. 7, pp. 868-879, 2014.
[215] S. Kärkkäinen, T. Heliö, P. Jääskeläinen et al., "Two novel mutations in the $\beta$-myosin heavy chain gene associated with dilated cardiomyopathy," European Journal of Heart Failure, vol. 6, no. 7, pp. 861-868, 2004.

[216] C. Meredith, R. Herrmann, C. Parry et al., "Mutations in the slow skeletal muscle fiber myosin heavy chain gene (MYH7) cause Laing early-onset distal myopathy (MPD1)," American Journal of Human Genetics, vol. 75, no. 4, pp. 703-708, 2004.

[217] B. Udd, "Genetics and pathogenesis of distal muscular dystrophies," Advances in Experimental Medicine and Biology, vol. 652, pp. 23-38, 2009.

[218] S. Waldmüller, P. Freund, S. Mauch, R. Toder, and H.-P. Vosberg, "Low-density DNA microarrays are versatile tools to screen for known mutations in hypertrophic cardiomyopathy," Human Mutation, vol. 19, no. 5, pp. 560-569, 2002.

[219] G. Tasca, E. Ricci, S. Penttilä et al., "New phenotype and pathology features in MYH7-related distal myopathy," Neuromuscular Disorders, vol. 22, no. 7, pp. 640-647, 2012.

[220] N. F. Clarke, K. Amburgey, J. Teener et al., "A novel mutation expands the genetic and clinical spectrum of MYH7-related myopathies," Neuromuscular Disorders, vol. 23, no. 5, pp. 432436, 2013.

[221] N. B. Romero, T. Xie, E. Malfatti et al., "Autosomal dominant eccentric core disease caused by a heterozygous mutation in the MYH7 gene," Journal of Neurology, Neurosurgery and Psychiatry, vol. 85, no. 10, pp. 1149-1152, 2014.

[222] S. Klaassen, S. Probst, E. Oechslin et al., "Mutations in sarcomere protein genes in left ventricular noncompaction," Circulation, vol. 117, no. 22, pp. 2893-2901, 2008.

[223] E. Blair, C. Redwood, M. de Jesus Oliveira et al., "Mutations of the light meromyosin domain of the beta-myosin heavy chain rod in hypertrophic cardiomyopathy," Circulation Research, vol. 90, no. 3, pp. 263-269, 2002.

[224] X. Stalpers, A. Verrips, J. Braakhekke, M. Lammens, A. van den Wijngaard, and A. Mostert, "Scoliosis surgery in a patient with 'de novo' myosin storage myopathy," Neuromuscular Disorders, vol. 21, no. 11, pp. 812-815, 2011.

[225] D. E. Dye, B. Azzarelli, H. H. Goebel, and N. G. Laing, "Novel slow-skeletal myosin (MYH7) mutation in the original myosin storage myopathy kindred," Neuromuscular Disorders, vol. 16, no. 6, pp. 357-360, 2006.

[226] T. Z. Armel and L. A. Leinwand, "Mutations in the $\beta$-myosin rod cause myosin storage myopathy via multiple mechanisms," Proceedings of the National Academy of Sciences of the United States of America, vol. 106, no. 15, pp. 6291-6296, 2009.

[227] H. Tajsharghi, L.-E. Thornell, C. Lindberg, B. Lindvall, K.G. Henriksson, and A. Oldfors, "Myosin storage myopathy associated with a heterozygous missense mutation in MYH7," Annals of Neurology, vol. 54, no. 4, pp. 494-500, 2003.

[228] J. Finsterer, O. Brandau, C. Stöllberger, W. Wallefeld, N. G. Laing, and F. Laccone, "Distal myosin heavy chain-7 myopathy due to the novel transition c.5566G>A (p.E1856K) with high interfamilial cardiac variability and putative anticipation," $\mathrm{Neu}$ romuscular Disorders, vol. 24, no. 8, pp. 721-725, 2014.

[229] H. Tajsharghi, A. Oldfors, D. P. Macleod, and M. Swash, "Homozygous mutation in MYH7 in myosin storage myopathy and cardiomyopathy," Neurology, vol. 68, no. 12, p. 962, 2007.

[230] S. Bohlega, S. N. Abu-Amero, S. M. Wakil et al., "Mutation of the slow myosin heavy chain rod domain underlies hyaline body myopathy," Neurology, vol. 62, no. 9, pp. 1518-1521, 2004. 
[231] A. V. Postma, K. van Engelen, J. van de Meerakker et al., "Mutations in the sarcomere gene MYH7 in Ebstein anomaly," Circulation: Cardiovascular Genetics, vol. 4, no. 1, pp. 43-50, 2011.

[232] S. Ortolano, R. Tarrío, P. Blanco-Arias et al., "A novel MYH7 mutation links congenital fiber type disproportion and myosin storage myopathy," Neuromuscular Disorders, vol. 21, no. 4, pp. 254-262, 2011.

[233] M. Koebis, N. Ohsawa, Y. Kino, N. Sasagawa, I. Nishino, and S. Ishiura, "Alternative splicing of myomesin 1 gene is aberrantly regulated in myotonic dystrophy type 1," Genes to Cells, vol. 16, no. 9, pp. 961-972, 2011.

[234] R. Siegert, A. Perrot, S. Keller et al., "A myomesin mutation associated with hypertrophic cardiomyopathy deteriorates dimerisation properties," Biochemical and Biophysical Research Communications, vol. 405, no. 3, pp. 473-479, 2011.

[235] T. Arimura, Y. Matsumoto, O. Okazaki et al., "Structural analysis of obscurin gene in hypertrophic cardiomyopathy," Biochemical and Biophysical Research Communications, vol. 362, no. 2, pp. 281-287, 2007.

[236] A. Evilä, A. Vihola, J. Sarparanta et al., "Atypical phenotypes in titinopathies explained by second titin mutations," Annals of Neurology, vol. 75, no. 2, pp. 230-240, 2014.

[237] J. R. Golbus, M. J. Puckelwartz, J. P. Fahrenbach, L. M. DellefaveCastillo, D. Wolfgeher, and E. M. McNally, "Population-based variation in cardiomyopathy genes," Circulation: Cardiovascular Genetics, vol. 5, no. 4, pp. 391-399, 2012.

[238] O. Ceyhan-Birsoy, P. B. Agrawal, C. Hidalgo et al., "Recessive truncating titin gene, TTN, mutations presenting as centronuclear myopathy," Neurology, vol. 81, no. 14, pp. 1205-1214, 2013.

[239] Y. Matsumoto, T. Hayashi, N. Inagaki et al., "Functional analysis of titin/connectin N2-B mutations found in cardiomyopathy," Journal of Muscle Research \& Cell Motility, vol. 26, no. 6-8, pp. 367-374, 2005.

[240] D. S. Herman, L. Lam, M. R. Taylor et al., "Truncations of titin causing dilated cardiomyopathy," The New England Journal of Medicine, vol. 366, no. 7, pp. 619-628, 2012.

[241] V. Carmignac, M. A. M. Salih, S. Quijano-Roy et al., "C-terminal titin deletions cause a novel early-onset myopathy with fatal cardiomyopathy," Annals of Neurology, vol. 61, no. 4, pp. 340351, 2007.

[242] M. Taylor, S. Graw, G. Sinagra et al., "Genetic variation in titin in arrhythmogenic right ventricular cardiomyopathy-overlap syndromes," Circulation, vol. 124, no. 8, pp. 876-885, 2011.

[243] P. Hackman, S. Marchand, J. Sarparanta et al., “Truncating mutations in C-terminal titin may cause more severe tibial muscular dystrophy (TMD)," Neuromuscular Disorders, vol. 18, no. 12, pp. 922-928, 2008.

[244] P. Hackman, A. Vihola, H. Haravuori et al., "Tibial muscular dystrophy is a titinopathy caused by mutations in TTN, the gene encoding the giant skeletal-muscle protein titin," American Journal of Human Genetics, vol. 71, no. 3, pp. 492-500, 2002.

[245] B. Udd, A. Vihola, J. Sarparanta, I. Richard, and P. Hackman, "Titinopathies and extension of the M-line mutation phenotype beyond distal myopathy and LGMD2J," Neurology, vol. 64, no. 4, pp. 636-642, 2005.

[246] M. Pollazzon, T. Suominen, S. Penttilä et al., "The first Italian family with tibial muscular dystrophy caused by a novel titin mutation," Journal of Neurology, vol. 257, no. 4, pp. 575-579, 2010.
[247] P. Y. K. van den Bergh, O. Bouquiaux, C. Verellen et al., “Tibial muscular dystrophy in a Belgian family," Annals of Neurology, vol. 54, no. 2, pp. 248-251, 2003.

[248] G. P. Comi, F. Fortunato, S. Lucchiari et al., " $\beta$-enolase deficiency, a new metabolic myopathy of distal glycolysis," Annals of Neurology, vol. 50, no. 2, pp. 202-207, 2001.

[249] M. Su, J. Wang, L. Kang et al., "Rare variants in genes encoding $M u R F 1$ and MuRF2 are modifiers of hypertrophic cardiomyopathy," International Journal of Molecular Sciences, vol. 15, no. 6, pp. 9302-9313, 2014.

[250] S. Tsujino, S. Servidei, P. Tonin, S. Shanske, G. Azan, and S. DiMauro, "Identification of three novel mutations in nonAshkenazi Italian patients with muscle phosphofructokinase deficiency," American Journal of Human Genetics, vol. 54, no. 5, pp. 812-819, 1994.

[251] J. B. Sherman, N. Raben, C. Nicastri et al., "Common mutations in the phosphofructokinase-M gene in Ashkenazi Jewish patients with glycogenesis VII-and their population frequency," The American Journal of Human Genetics, vol. 55, no. 2, pp. 305-313, 1994.

[252] O. Musumeci, C. Bruno, T. Mongini et al., "Clinical features and new molecular findings in muscle phosphofructokinase deficiency (GSD type VII)," Neuromuscular Disorders, vol. 22, no. 4, pp. 325-330, 2012.

[253] O. Vasconcelos, K. Sivakumar, M. C. Dalakas et al., "Nonsense mutation in the phosphofructokinase muscle subunit gene associated with retention of intron 10 in one of the isolated transcripts in Ashkenazi Jewish patients with Tarui disease," Proceedings of the National Academy of Sciences of the United States of America, vol. 92, no. 22, pp. 10322-10326, 1995.

[254] N. Raben, R. Exelbert, R. Spiegel et al., "Functional expression of human mutant phosphofructokinase in yeast: genetic defects in French Canadian and Swiss patients with phosphofructokinase deficiency," American Journal of Human Genetics, vol. 56, no. 1, pp. 131-141, 1995.

[255] J.-L. Vives-Corrons, P. Koralkova, J. M. Grau, M. D. M. M. Pereira, and R. Van Wijk, "First description of phosphofructokinase deficiency in spain: identification of a novel homozygous missense mutation in the PFKM gene," Frontiers in Physiology, vol. 4, article 393, 2013.

[256] H. Nakajima, N. Raben, T. Hamaguchi, and T. Yamasaki, "Phosphofructokinase deficiency; past, present and future," Current Molecular Medicine, vol. 2, no. 2, pp. 197-212, 2002.

[257] R. C. Nichols, O. Rudolphi, B. Ek, R. Exelbert, P. H. Plotz, and N. Raben, "Glycogenosis type VII (Tarui disease) in a Swedish family: two novel mutations in muscle phosphofructokinase gene (PFK-M) resulting in intron retentions," American Journal of Human Genetics, vol. 59, no. 1, pp. 59-65, 1996.

[258] T. Hamaguchi, H. Nakajima, T. Noguchi et al., "A new variant of muscle phosphofructokinase deficiency in a Japanese case with abnormal RNA splicing," Biochemical and Biophysical Research Communications, vol. 202, no. 1, pp. 444-449, 1994.

[259] H. Tajsharghi and A. Oldfors, "Myosinopathies: pathology and mechanisms," Acta Neuropathologica, vol. 125, no. 1, pp. 3-18, 2013.

[260] B. Udd, "Third filament diseases," Advances in Experimental Medicine and Biology, vol. 642, pp. 99-115, 2008.

[261] M. A. Ackermann, L. Y. Hu, and A. Kontrogianni-Konstnatopoulos, "Intercellular connections in the heart: the intercalated disc," in Cardiomyopathies-From Basic Research to Clinical Management, J. Veselka, Ed., InTech, 2012. 
[262] M. A. Ackermann, P. D. Patel, J. Valenti et al., "Loss of actomyosin regulation in distal arthrogryposis myopathy due to mutant myosin binding protein-C slow," The FASEB Journal, vol. 27, no. 8, pp. 3217-3228, 2013.

[263] M. A. Ackermann and A. Kontrogianni-Konstantopoulos, "Myosin binding protein-C slow: a multifaceted family of proteins with a complex expression profile in fast and slow twitch skeletal muscles," Frontiers in Physiology, vol. 4, article 391, 2013.

[264] W. Piyamongkol, J. C. Harper, J. K. Sherlock et al., "A successful strategy for preimplantation genetic diagnosis of myotonic dystrophy using multiplex fluorescent PCR," Prenatal Diagnosis, vol. 21, no. 3, pp. 223-232, 2001.

[265] I. Agarkova, D. Auerbach, E. Ehler, and J.-C. Perriard, "A novel marker for vertebrate embryonic heart, the EH-myomesin isoform," The Journal of Biological Chemistry, vol. 275, no. 14, pp. 10256-10264, 2000.

[266] R. Schoenauer, M. Y. Emmert, A. Felley et al., "EH-myomesin splice isoform is a novel marker for dilated cardiomyopathy," Basic Research in Cardiology, vol. 106, no. 2, pp. 233-247, 2011.

[267] T. Shathasivam, T. Kislinger, and A. O. Gramolini, "Genes, proteins and complexes: the multifaceted nature of FHL family proteins in diverse tissues," Journal of Cellular and Molecular Medicine, vol. 14, no. 12, pp. 2702-2720, 2010.

[268] E. Malfatti, M. Olivé, A. L. Taratuto et al., "Skeletal muscle biopsy analysis in reducing body myopathy and other FHL1related disorders," Journal of Neuropathology \& Experimental Neurology, vol. 72, no. 9, pp. 833-845, 2013.

[269] J. S. Binder, F. Weidemann, B. Schoser et al., "Spongious hypertrophic cardiomyopathy in patients with mutations in the fourand-a-half LIM domain 1 gene," Circulation: Cardiovascular Genetics, vol. 5, no. 5, pp. 490-502, 2012.

[270] D.-S. Lim, R. Roberts, and A. J. Marian, "Expression profiling of cardiac genes in human hypertrophic cardiomyopathy: insight into the pathogenesis of phenotypes," Journal of the American College of Cardiology, vol. 38, no. 4, pp. 1175-1180, 2001.

[271] J. Yang, C. S. Moravec, M. A. Sussman et al., "Decreased SLIM1 expression and increased gelsolin expression in failing human hearts measured by high-density oligonucleotide arrays," Circulation, vol. 102, no. 25, pp. 3046-3052, 2000.

[272] B. R. Wilding, M. J. McGrath, G. Bonne, and C. A. Mitchell, "FHL1 mutants that cause clinically distinct human myopathies form protein aggregates and impair myoblast differentiation," Journal of Cell Science, vol. 127, no. 10, pp. 2269-2281, 2014.

[273] C. Angelini, "Spectrum of metabolic myopathies," Biochimica et Biophysica Acta, vol. 1852, no. 4, pp. 615-621, 2015.

[274] H. Morisaki, I. Higuchi, M. Abe, M. Osame, and T. Morisaki, "First missense mutations (R388W and R425H) of AMPD1 accompanied with myopathy found in a Japanese patient," Human Mutation, vol. 16, no. 6, pp. 467-472, 2000.

[275] T. Morisaki, M. Gross, H. Morisaki, D. Pongratz, N. Zollner, and E. W. Holmes, "Molecular basis of AMP deaminase deficiency in skeletal muscle," Proceedings of the National Academy of Sciences of the United States of America, vol. 89, no. 14, pp. 6457-6461, 1992.

[276] M. Gross, E. Rötzer, P. Kölle et al., "A G468-T AMPD1 mutant allele contributes to the high incidence of myoadenylate deaminase deficiency in the Caucasian population," Neuromuscular Disorders, vol. 12, no. 6, pp. 558-565, 2002.

[277] P. J. Isackson, H. Bujnicki, C. O. Harding, and G. D. Vladutiu, "Myoadenylate deaminase deficiency caused by alternative splicing due to a novel intronic mutation in the AMPD1 gene," Molecular Genetics and Metabolism, vol. 86, no. 1-2, pp. 250-256, 2005.

[278] D. K. Mahnke-Zizelman and R. L. Sabina, "Cloning of human AMP deaminase isoform E cDNAs. Evidence for a third AMPD gene exhibiting alternatively spliced $5^{\prime}$-exons," Journal of Biological Chemistry, vol. 267, no. 29, pp. 20866-20877, 1992.

[279] C. Suetta, U. Frandsen, L. Jensen et al., "Aging affects the transcriptional regulation of human skeletal muscle disuse atrophy," PLoS ONE, vol. 7, no. 12, Article ID e51238, 2012.

[280] M. Merli, M. Giusto, A. Molfino et al., "MuRF-1 and p-GSK3 $\beta$ expression in muscle atrophy of cirrhosis," Liver International, vol. 33, no. 5, pp. 714-721, 2013.

[281] S. Gielen, M. Sandri, I. Kozarez et al., "Exercise training attenuates MuRF-1 expression in the skeletal muscle of patients with chronic heart failure independent of age: the randomized leipzig exercise intervention in chronic heart failure and aging catabolism study," Circulation, vol. 125, no. 22, pp. 2716-2727, 2012.

[282] O. Mayans and S. Labeit, "MuRFs: specialized members of the TRIM/RBCC family with roles in the regulation of the trophic state of muscle and its metabolism," Advances in Experimental Medicine and Biology, vol. 770, pp. 119-129, 2012. 

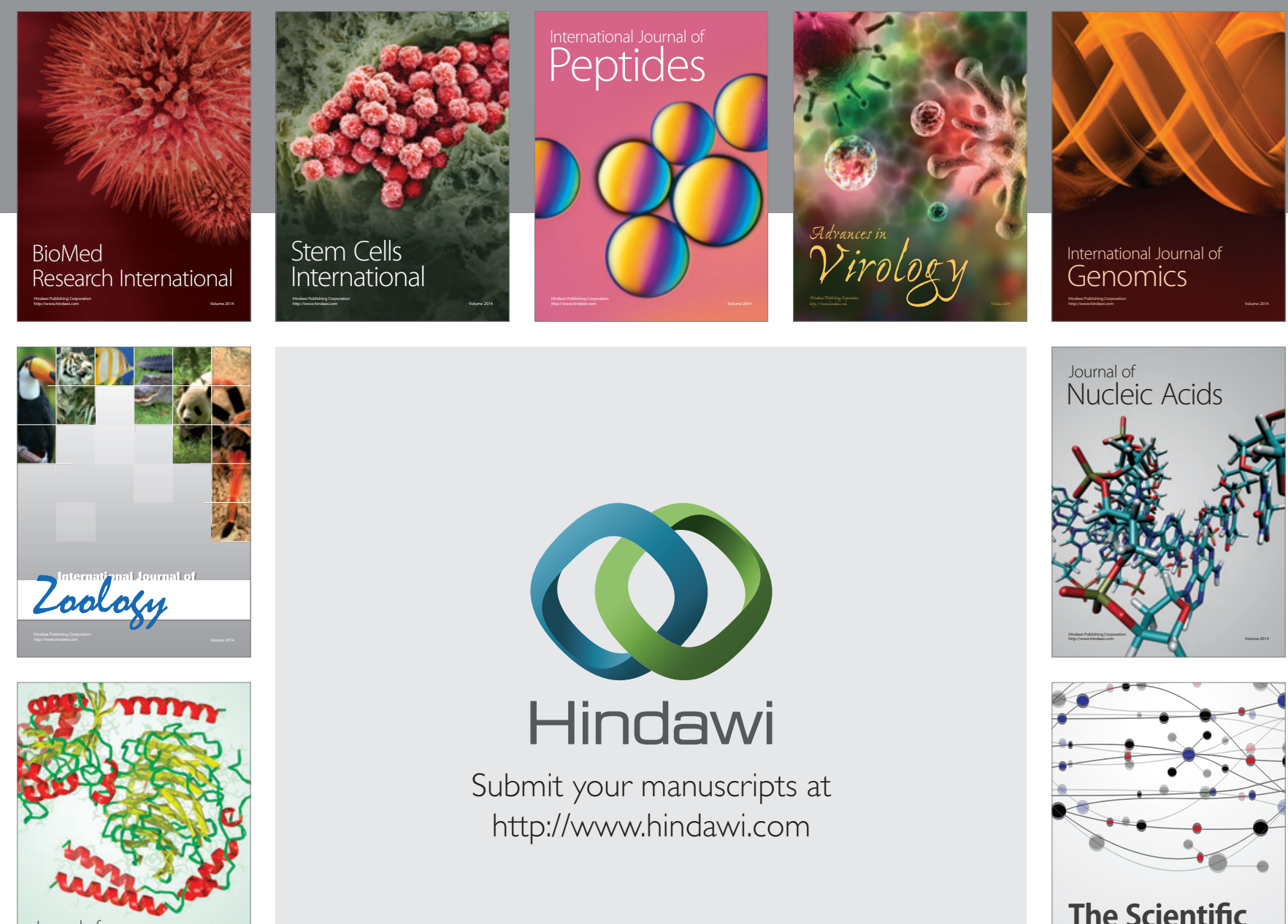

Submit your manuscripts at

http://www.hindawi.com

Journal of
Signal Transduction
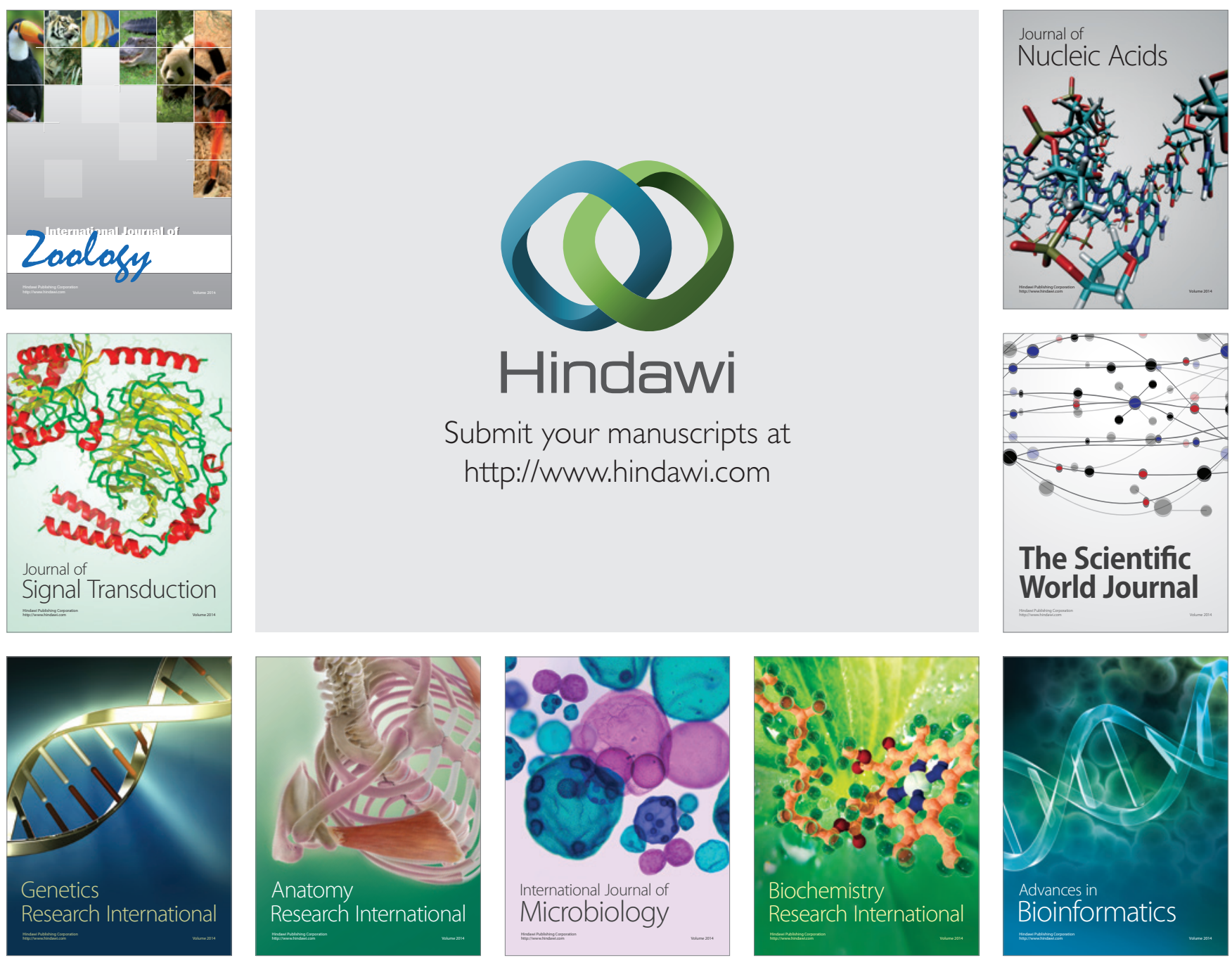

The Scientific World Journal
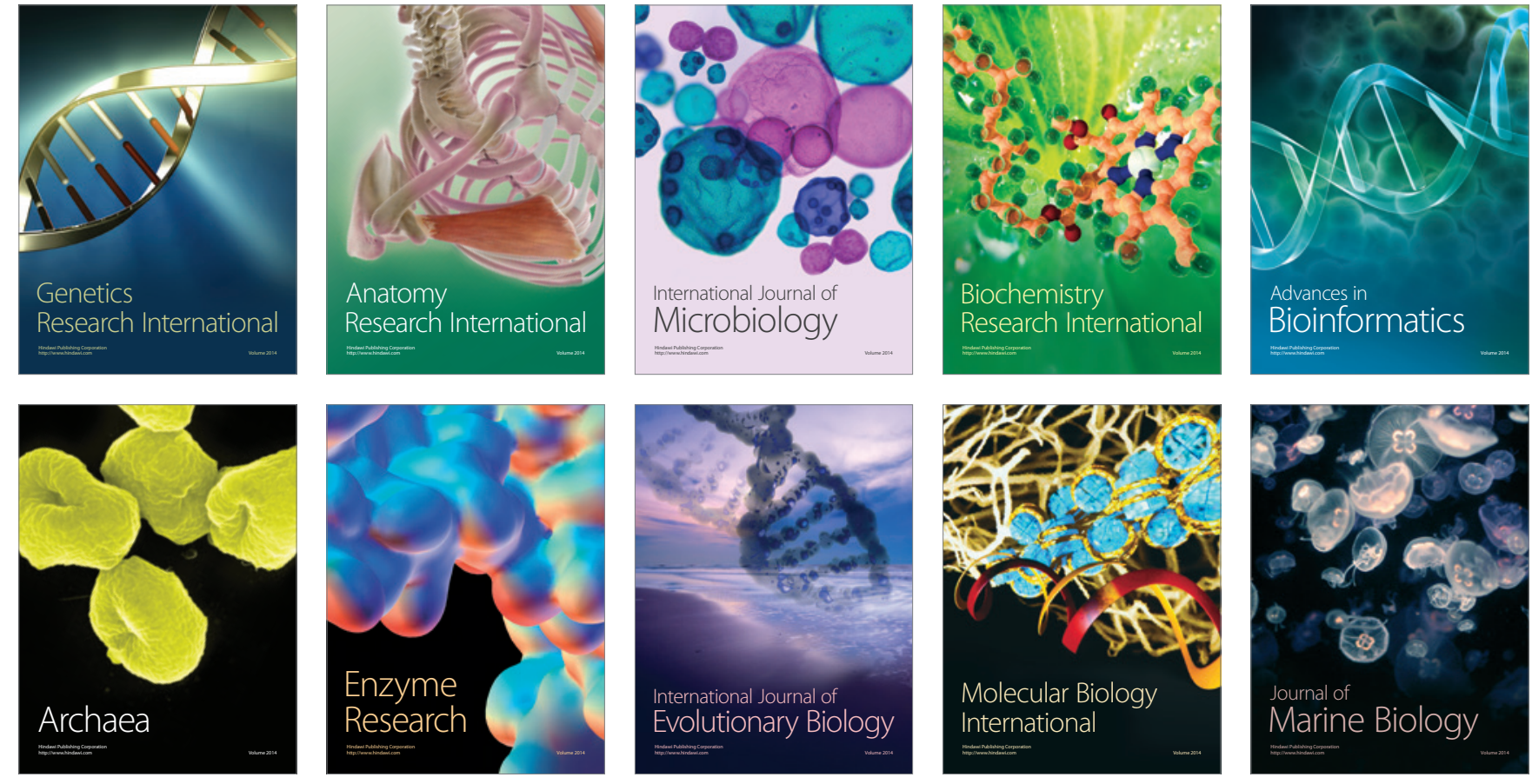